MATHEMATICS OF COMPUTATION

Volume 78, Number 266, April 2009, Pages 999-1029

S $0025-5718(08) 02156-\mathrm{X}$

Article electronically published on August 27, 2008

\title{
DISCRETE FOURIER ANALYSIS ON A DODECAHEDRON AND A TETRAHEDRON
}

\author{
HUIYUAN LI AND YUAN XU
}

\begin{abstract}
A discrete Fourier analysis on the dodecahedron is studied, from which results on a tetrahedron are deduced by invariance. The results include Fourier analysis in trigonometric functions, interpolation and cubature formulas on these domains. In particular, a trigonometric Lagrange interpolation on the tetrahedron is shown to satisfy an explicit compact formula and the Lebesgue constant of the interpolation is shown to be in the order of $(\log n)^{3}$.
\end{abstract}

\section{INTRODUCTION}

It is well known that Fourier analysis in several variables can be developed based on the periodicity defined by a lattice, which is a discrete subgroup defined by $A \mathbb{Z}^{d}$, where $A$ is a nonsingular $d \times d$ matrix. A lattice $L:=A \mathbb{Z}^{d}$ is called a tiling lattice of $\mathbb{R}^{d}$ if there is bounded set $\Omega$ that tiles $\mathbb{R}^{d}$ in the sense that $\Omega+L=\mathbb{R}^{d}$. Let $L$ be a tiling lattice and $L^{\perp}:=A^{-\operatorname{tr}} \mathbb{Z}^{d}$ its dual lattice; then a theorem of Fuglede 5 states that the family of exponentials $\left\{e^{2 \pi i \alpha \cdot x}: \alpha \in L^{\perp}\right\}$ forms an orthonormal basis for $L^{2}(\Omega)$. The Fourier expansion on $\Omega$ is essentially the usual multivariate Fourier series under a change of variables $x \mapsto A^{-1} x$.

One can also develop a discrete Fourier analysis associated with a lattice, starting with a discrete Fourier transform based on $L^{\perp}$, which has applications in areas such as signal processing and sampling theory (see, for example, [3, 17, 10]). Recently in 9, we studied the discrete Fourier transform and used it to derive results on cubature and trigonometric interpolation on the domain $\Omega$, both are important tools in numerical computation and approximation theory. The simplest domain for the tiling lattice is the regular hexagon, which has the invariance of the reflection group $\mathcal{A}_{2}$. The fundamental domain of the hexagon under $\mathcal{A}_{2}$ is an equilateral triangle. A detailed study of the discrete Fourier analysis is carried out on the hexagon and on the triangle in 9. The invariant and the anti-invariant projections of the basic exponential functions are analogues of cosine and sine functions on the triangle, which have been studied previously in [8, 11]. Explicit and compact formulas are derived for several cubature formulas and interpolation functions in [9]. In particular, we found a compact formula for the Lagrange interpolation by trigonometric functions that interpolates at $X_{n}:=\left\{\left(\frac{i}{n}, \frac{j}{n}\right): 0 \leq i \leq j \leq n\right\}$ on the

Received by the editor April 10, 2007 and, in revised form, February 29, 2008.

2000 Mathematics Subject Classification. Primary 41A05, 41A10.

Key words and phrases. Discrete Fourier series, trigonometric, Lagrange interpolation, dodecahedron, tetrahedron.

The first authors were supported by NSFC Grants 10601056, 10431050 and 60573023 . The second author was supported by NSF Grant DMS-0604056. 
triangle $T:=\{(x, y): x, y \geq 0, x+y \leq 1\}$ and proved that its Lebesgue constant is in the order of $(\log n)^{2}$. The result on interpolation is noteworthy since it is in sharp contract to the algebraic polynomial interpolation on $X_{n}$, which has an undesirable convergence behavior.

The purpose of the present paper is to carry out a similar analysis on $\mathbb{R}^{3}$ for a tetrahedron, also called a simplex in $\mathbb{R}^{3}$. For this we work with the face-centered cubic (fcc) lattice, which has the symmetry of reflection group $\mathcal{A}_{3}$. The domain that tiles $\mathbb{R}^{3}$ with the fcc lattice is the rhombic dodecahedron (see Figure 3.2), whose fundamental domain under $\mathcal{A}_{3}$ is a regular tetrahedron. We shall develop in detail a Fourier analysis on these two domains, study analogues of cosine and sine functions as in the case of hexagon, and establish compact formulas for discrete inner product, cubature formulas and Dirichlet kernels.

Just as in the case of the regular hexagon [9, 11, the analysis on the rhombic dodecahedron and the tetrahedron is carried out using homogeneous coordinates of $\mathbb{R}^{4}$ instead of in $\mathbb{R}^{3}$. This has the advantage that our formulas are more symmetric and the symmetry of the domain becomes more transparent. The Fourier transform on the dodecahedron as well as the generalized cosine and sine functions were studied earlier in 12 using a homogeneous coordinate system in $\mathbb{R}^{6}$. We choose our homogeneous coordinates in $\mathbb{R}^{4}$ since $\mathcal{A}_{3}$ can be regarded as a permutation group on four elements.

It should be pointed out that the development on a specific domain does not follow immediately from the general theory. To tile the space without overlap, the domain $\Omega$ can only include part of its boundary. For the discrete Fourier analysis, this fact causes a loss of symmetry; for example, for the rhombic dodecahedron, the discrete Fourier transform is defined using only part of the boundary points. In order to obtain results that are symmetric, we have to modify definitions to include all boundary points, which can be delicate if the orthogonality is to be preserved. The difficulty lies in the congruent relations of the boundary. In order to understand the periodicity based on the rhombic dodecahedron, we need to understand the congruence of the boundary under translation by the lattice. Furthermore, in order to transform results from the rhombic dodecahedron to the tetrahedron, we need to understand the action of $\mathcal{A}_{3}$ on the boundary. The complication of the congruence of the boundary is also one of the main reasons why we restrict ourself to $\mathbb{R}^{3}$ instead of dealing with lattices on $\mathbb{R}^{d}$ that are invariant under $\mathcal{A}_{d}$ (see [2]) for all $d \geq 3$.

One of our main results is a compact formula for the Lagrange interpolation based on the regular points on the tetrahedron, whose Lebesgue constant is shown to be in the order of $(\log n)^{3}$. Again, this is a result in sharp contrast to interpolation by algebraic polynomials on the same set of points. Interpolation by simple functions is an important tool in numerical analysis that has a variety of applications. For interpolation on the point sets in several variables, little results are known if the point sets are not of tensor product type. Moreover, most studies consider mainly interpolation by algebraic polynomials, which face the problem of choosing interpolation points, as equally spaced points do not yield favorable results [1. Our study in [9] and in the present paper demonstrates that interpolation at equally spaced points on the triangle and on the tetrahedron can be solved with trigonometric functions: the interpolation can be carried out by compact formulas that offer fast computation, and the convergence behavior is as good as can be expected since a Lebesgue constant of $(\log n)^{d}$ for interpolation in $\mathbb{R}^{d}$ is about 
optimal. In the present paper, we concentrate on a theoretic framework that leads to trigonometric interpolation on the tetrahedron; a numerical study is for a future work.

The paper is organized as follows. In Section 2 we sum up results from the general theory of discrete Fourier analysis associated with lattice. The analysis on the rhombic dodecahedron will be carried out in Section 3, including a detailed study on the congruence of the boundary. Results on the tetrahedron are developed in Section 4, including generalized sine and cosine functions.

\section{Discrete Fourier analysis With Lattice}

In this section we recall results on discrete Fourier analysis associated with lattice. Background and the content of the first subsection can be found in [2, 3, 17, 10, Results in the second subsection are developed in 9 . We shall be brief and refer the proof and discussions to the above mentioned references.

2.1. Lattice and Fourier series. A lattice $L$ of $\mathbb{R}^{d}$ is a discrete subgroup that contains $d$ linearly independent vectors,

$$
L:=\left\{k_{1} a_{1}+k_{2} a_{2}+\cdots+k_{d} a_{d}: k_{i} \in \mathbb{Z}, i=1,2, \ldots, d\right\},
$$

where $a_{1}, \ldots, a_{d}$ are linearly independent column vectors in $\mathbb{R}^{d}$. Let $A \in \mathbb{R}^{d \times d}$ be the matrix whose columns are $a_{1}, \ldots, a_{d}$. Then $A$ is called a generator matrix of the lattice $L$. We can write $L$ as $L_{A}$ and a short notation for $L_{A}$ is $A \mathbb{Z}^{d}$; that is,

$$
L_{A}=A \mathbb{Z}^{d}=\left\{A k: k \in \mathbb{Z}^{d}\right\} .
$$

Throughout this paper, we shall treat a vector in the Euclidean space as a column vector whenever needed. As a result, $x \cdot y=x^{\text {tr }} y$, where $x^{\text {tr }}$ denotes the transpose of $x$. The dual lattice $L^{\perp}$ of $L$ is given by

$$
L^{\perp}:=\left\{x \in \mathbb{R}^{d}: x \cdot y \in \mathbb{Z} \text { for all } y \in L\right\},
$$

where $x \cdot y$ denotes the usual Euclidean inner product of $x$ and $y$. The generator matrix of $L^{\perp}$ is $A^{-\mathrm{tr}}$.

A bounded set $\Omega \subset \mathbb{R}^{d}$ is said to tile $\mathbb{R}^{d}$ with the lattice $L$ if

$$
\sum_{\alpha \in L} \chi_{\Omega}(x+\alpha)=1, \quad \text { for almost all } x \in \mathbb{R}^{d},
$$

where $\chi_{\Omega}$ denotes the characteristic function of $\Omega$, which we write as $\Omega+L=\mathbb{R}^{d}$. Tiling and Fourier analysis are closely related as demonstrated by the Fuglede theorem. Let $\int_{\Omega} f(x) d x$ denote the integration of the function $f$ over $\Omega$. Let $\langle\cdot, \cdot\rangle_{\Omega}$ denote the inner product in $L^{2}(\Omega)$,

$$
\langle f, g\rangle_{\Omega}:=\frac{1}{|\Omega|} \int_{\Omega} f(x) \overline{g(x)} d x,
$$

where $|\Omega|$ denotes the measure of $\Omega$. The following fundamental result was proved by Fuglede in 5 .

Theorem 2.1. Let $\Omega \subset \mathbb{R}^{d}$ be a bounded domain and let $L$ be a lattice of $\mathbb{R}^{d}$. Then $\Omega+L=\mathbb{R}^{d}$ if and only if $\left\{e^{2 \pi i \alpha \cdot x}: \alpha \in L^{\perp}\right\}$ is an orthonormal basis with respect to the inner product (2.1). 
The orthonormal property is defined with respect to the normalized Lebesgue measure on $\Omega$. If $L=L_{A}$, then the measure of $\Omega$ is equal to $\sqrt{\operatorname{det}\left(A^{\operatorname{tr}} A\right)}$. Furthermore, we can write $\alpha \in L_{A}^{\perp}=A^{-\operatorname{tr}} \mathbb{Z}^{d}$ as $\alpha=A^{- \text {tr }} k$ with $k \in \mathbb{Z}^{d}$, so that $\alpha \cdot x=k^{\operatorname{tr}} A^{-1} x$. Hence the orthogonality in the theorem is

$$
\frac{1}{\sqrt{\operatorname{det}\left(A^{\operatorname{tr} A)}\right.}} \int_{\Omega} \mathrm{e}^{2 \pi i k^{\mathrm{tr}} A^{-1} x} d x=\delta_{k, 0}, \quad k \in \mathbb{Z}^{d} .
$$

The set $\Omega$ is called a spectral set (fundamental region) for the lattice $L$. If $L=L_{A}$ we also write $\Omega=\Omega_{A}$.

A function $f \in L^{1}\left(\Omega_{A}\right)$ can be expanded into a Fourier series

$$
f(x) \sim \sum_{k \in \mathbb{Z}^{d}} c_{k} \mathrm{e}^{2 \pi i k^{\mathrm{tr}} A^{-1} x}, \quad c_{k}=\frac{1}{\sqrt{\operatorname{det}\left(A^{\operatorname{tr} A}\right)}} \int_{\Omega} f(x) \mathrm{e}^{-2 \pi i k^{\mathrm{tr}} A^{-1} x} d x .
$$

The Fourier transform $\widehat{f}$ of a function defined on $L^{1}\left(\mathbb{R}^{d}\right)$ and its inversion are defined by

$$
\widehat{f}(\xi)=\int_{\mathbb{R}^{d}} f(x) \mathrm{e}^{-2 \pi i \xi \cdot x} d x, \quad f(x)=\int_{\mathbb{R}^{d}} \widehat{f}(\xi) \mathrm{e}^{2 \pi i \xi \cdot x} d \xi .
$$

Our first result is the following sampling theorem (see, for example, [7, 10]).

Proposition 2.2. Let $\Omega$ be the spectral set of the lattice $A \mathbb{Z}^{d}$. Assume that $\widehat{f}$ is supported on $\Omega$ and $\widehat{f} \in L^{2}(\Omega)$. Then

$$
f(x)=\sum_{k \in \mathbb{Z}^{d}} f\left(A^{-\operatorname{tr}} k\right) \Phi_{\Omega}\left(x-A^{-\operatorname{tr}} k\right)
$$

in $L^{2}(\Omega)$, where

$$
\Phi_{\Omega}(x)=\frac{1}{\sqrt{\operatorname{det}\left(A^{\operatorname{tr} A}\right)}} \int_{\Omega} \mathrm{e}^{2 \pi i \xi \cdot x} d \xi .
$$

This theorem is a consequence of the Poisson summation formula. We notice that

$$
\Phi_{\Omega}\left(A^{-\operatorname{tr}} j\right)=\delta_{0, j}, \quad \text { for all } j \in \mathbb{Z}^{d},
$$

by Theorem 2.1, so that $\Phi_{\Omega}$ can be considered as a cardinal interpolation function.

2.2. Discrete Fourier analysis and interpolation. A function $f$ defined on $\mathbb{R}^{d}$ is called periodic with respect to the lattice $A \mathbb{Z}^{d}$ if

$$
f(x+A k)=f(x) \quad \text { for all } k \in \mathbb{Z}^{d} .
$$

The spectral set $\Omega$ of the lattice $A \mathbb{Z}^{d}$ is not unique. In order to carry out the discrete Fourier analysis with respect to the lattice, we shall fix $\Omega$ such that $\Omega$ contains 0 in its interior and we further require that $\Omega$ tiles $\mathbb{R}^{d}$ with $L_{A}$ without overlapping and without gap. In other words, we require that

$$
\sum_{k \in \mathbb{Z}^{d}} \chi_{\Omega}(x+A k)=1, \quad \text { for all } x \in \mathbb{R}^{d} .
$$

For example, we can take $\Omega=\left[-\frac{1}{2}, \frac{1}{2}\right)^{d}$ for the standard cubic lattice $\mathbb{Z}^{d}$.

Definition 2.3. Let $A$ and $B$ be two nonsingular matrices in $\mathbb{R}^{d \times d}, \Omega_{A}$ and $\Omega_{B}$ satisfy (2.3). Assume all entries of $N:=B^{\mathrm{tr}} A$ are integers. Define

$$
\Lambda_{N}:=\left\{k \in \mathbb{Z}^{d}: B^{-\operatorname{tr}} k \in \Omega_{A}\right\} \text { and } \Lambda_{N}^{\dagger}:=\left\{k \in \mathbb{Z}^{d}: A^{-\operatorname{tr}} k \in \Omega_{B}\right\} .
$$


Two points $x, y \in \mathbb{R}^{d}$ are said to be congruent with respect to the lattice $A \mathbb{Z}^{d}$, if $x-y \in A \mathbb{Z}^{d}$, and we write $x \equiv y(\bmod A)$. The following two theorems are the central results for the discrete Fourier transform.

Theorem 2.4. Let $A, B$ and $N$ be as in Definition 2.3 . Then

$$
\frac{1}{|\operatorname{det}(N)|} \sum_{j \in \Lambda_{N}} \mathrm{e}^{2 \pi i k^{\mathrm{tr}} N^{-1} j}= \begin{cases}1, & \text { if } k \equiv 0 \quad\left(\bmod N^{\mathrm{tr}}\right), \\ 0, & \text { otherwise. }\end{cases}
$$

Theorem 2.5. Let $A, B$ and $N$ be as in Definition 2.3. Define the discrete inner product

$$
\langle f, g\rangle_{N}=\frac{1}{|\operatorname{det}(N)|} \sum_{j \in \Lambda_{N}} f\left(B^{-\operatorname{tr}} j\right) \overline{g\left(B^{-\operatorname{tr}} j\right)}
$$

for $f, g \in C\left(\Omega_{A}\right)$, the space of continuous functions on $\Omega_{A}$. Then

$$
\langle f, g\rangle_{N}=\langle f, g\rangle
$$

for all $f, g$ in the finite dimensional subspace

$$
\mathcal{H}_{N}:=\operatorname{span}\left\{\phi_{k}: \phi_{k}(x)=\mathrm{e}^{2 \pi i k^{\mathrm{tr}} A^{-1} x}, k \in \Lambda_{N}^{\dagger}\right\}
$$

Let $|E|$ denote the cardinality of the set $E$. Then the dimension of $\mathcal{H}_{N}$ is $\left|\Lambda_{N}^{\dagger}\right|$.

Let $\mathcal{I}_{N} f$ denote the Fourier expansion of $f \in C\left(\Omega_{A}\right)$ in $\mathcal{H}_{N}$ with respect to the inner product $\langle\cdot, \cdot\rangle_{N}$. Then, analogous to the sampling theorem in Proposition 2.2 . $\mathcal{I}_{N} f$ satisfies the formula

$$
\mathcal{I}_{N} f(x)=\sum_{j \in \Lambda_{N}} f\left(B^{-\operatorname{tr}} j\right) \Phi_{\Omega_{B}}^{A}\left(x-B^{-\operatorname{tr}} j\right), \quad f \in C\left(\Omega_{A}\right),
$$

where

$$
\Phi_{\Omega_{B}}^{A}(x)=\frac{1}{|\operatorname{det}(N)|} \sum_{k \in \Lambda_{N}^{\dagger}} \mathrm{e}^{2 \pi i k^{\mathrm{tr}} A^{-1} x} .
$$

The following theorem shows that $\mathcal{I}_{N} f$ is an interpolation function.

Theorem 2.6. Let $A, B$ and $N$ be as in Definition 2.3. If $\Lambda_{N}^{\dagger}=\Lambda_{N^{\mathrm{tr}}}$, then $\mathcal{I}_{N} f$ is the unique interpolation operator on $N$ in $\mathcal{H}_{N}$; that is,

$$
\mathcal{I}_{N} f\left(B^{-\operatorname{tr}} j\right)=f\left(B^{-\operatorname{tr}} j\right), \quad \forall j \in \Lambda_{N}
$$

In particular, $\left|\Lambda_{N}\right|=\left|\Lambda_{N}^{\dagger}\right|$. Furthermore, the fundamental interpolation function $\Phi_{\Omega_{B}}^{A}$ satisfies

$$
\Phi_{\Omega_{B}}^{A}(x)=\sum_{k \in \mathbb{Z}^{d}} \Phi_{\Omega_{B}}(x+A k) .
$$

The above results have been used to develop a discrete Fourier analysis on a hexagon in 9]. In the following section, we apply it to the rhombic dodecahedron.

\section{Discrete Fourier anAlysis on the Rhombic DOdeCAHEDRon}

In this section we develop a discrete Fourier analysis on the rhombic dodecahedron. It contains five subsections. 


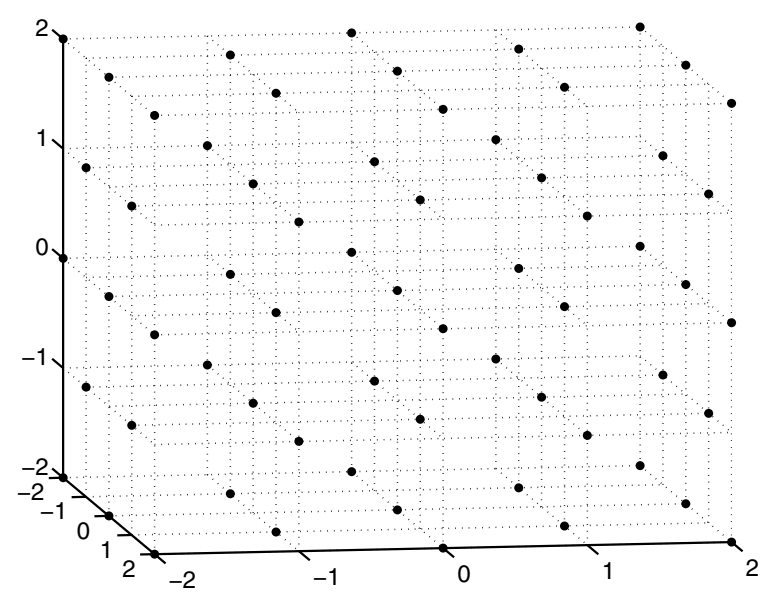

Figure 3.1. Face-centered cubic (fcc) lattice.

3.1. Face-centered cubic lattice and Fourier analysis. We consider the facecentered cubic (fcc) lattice given in Figure 3.1.

Just like the hexagon lattice, the fcc lattice offers the densest packing of $\mathbb{R}^{3}$ with unit balls, which is the so-called Kepler's conjecture and was proved recently in 6].

The generator matrix $A$ of fcc lattice is given by

$$
A=\left(\begin{array}{lll}
0 & 1 & 1 \\
1 & 0 & 1 \\
1 & 1 & 0
\end{array}\right) .
$$

The domain that tiles $\mathbb{R}^{3}$ with fcc lattice is the rhombic dodecahedron (see Figure $3.2)$. Thus, the spectral set of fcc lattice is

$$
\Omega=\left\{x \in \mathbb{R}^{3}:-1<x_{j} \pm x_{i} \leq 1,1 \leq i<j \leq 3\right\} .
$$

The strict inequality in the definition of $\Omega$ reflects our requirement that the tiling of the spectral set has no overlapping.

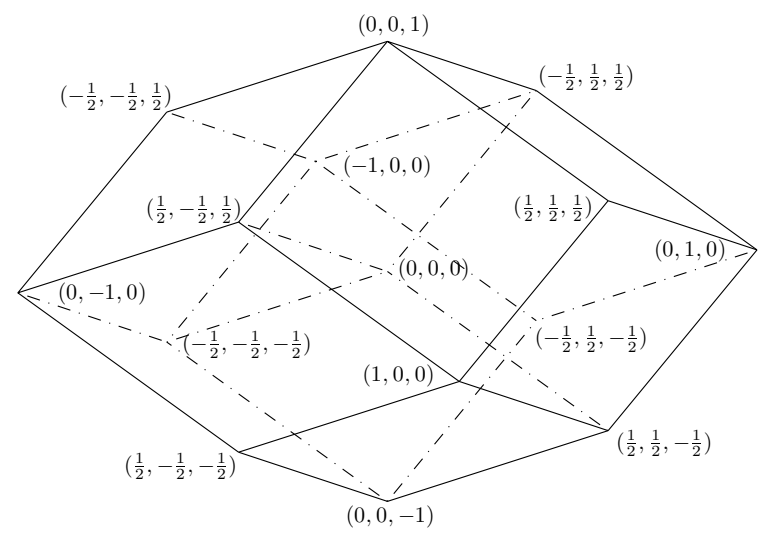

FIGURE 3.2. Rhombic dodecahedron 
Motivated by the study of [9, 11, 12, we shall use homogeneous coordinates $\mathbf{t}=\left(t_{1}, t_{2}, t_{3}, t_{4}\right)$, where $t_{1}+t_{2}+t_{3}+t_{4}=0$, in $\mathbb{R}^{4}$ for our analysis on the rhombic dodecahedron in $\mathbb{R}^{3}$. The advantage is that our formulas become more symmetric and the symmetry becomes more transparent under the homogeneous coordinates. Throughout the rest of this paper, we adopt the convention of using bold letters, such as $\mathbf{t}$, to denote the points in the space

$$
\mathbb{R}_{H}^{4}:=\left\{\mathbf{t}=\left(t_{1}, t_{2}, t_{3}, t_{4}\right) \in \mathbb{R}^{4}: t_{1}+t_{2}+t_{3}+t_{4}=0\right\} .
$$

In other words, the bold letters such as $\mathbf{t}$ and $\mathbf{k}$ will always mean homogeneous coordinates. The transformation between $x \in \mathbb{R}^{3}$ and $\mathbf{t} \in \mathbb{R}_{H}^{4}$ is defined by

$$
x=A\left(\begin{array}{l}
t_{1} \\
t_{2} \\
t_{3}
\end{array}\right) \quad \Longleftrightarrow\left\{\begin{array}{l}
x_{1}=t_{2}+t_{3}, \\
x_{2}=t_{1}+t_{3}, \\
x_{3}=t_{2}+t_{1},
\end{array}\right.
$$

and $t_{4}=-t_{1}-t_{2}-t_{3}$. Let us denote by $H$ and $U$ the matrices

$$
H=\left(\begin{array}{ccc}
1 & 0 & 0 \\
0 & 1 & 0 \\
0 & 0 & 1 \\
-1 & -1 & -1
\end{array}\right) \quad \text { and } U=\frac{1}{2}\left(\begin{array}{ccc}
-1 & 1 & 1 \\
1 & -1 & 1 \\
1 & 1 & -1 \\
-1 & -1 & -1
\end{array}\right)
$$

respectively. The columns of the matrix $U$ are orthonormal and $U^{\operatorname{tr}} U=I$. We then have $A=U^{\text {tr }} H$ and the inverse transform of (3.1) is

$$
\mathbf{t}=U x \quad \Longleftrightarrow \quad\left\{\begin{array}{l}
t_{1}=\frac{1}{2}\left(-x_{1}+x_{2}+x_{3}\right), \\
t_{2}=\frac{1}{2}\left(x_{1}-x_{2}+x_{3}\right), \\
t_{3}=\frac{1}{2}\left(x_{1}+x_{2}-x_{3}\right), \\
t_{4}=\frac{1}{2}\left(-x_{1}-x_{2}-x_{3}\right) .
\end{array}\right.
$$

In the homogenous coordinates, the spectral set is $\Omega_{H}:=\{\mathbf{t}=U x: x \in \Omega\}$ which, upon using (3.2), gives

$$
\Omega_{H}=\left\{\mathbf{t} \in \mathbb{R}_{H}^{4}:-1<t_{i}-t_{j} \leq 1,1 \leq i<j \leq 4\right\} .
$$

Figure 3.3 shows again the rhombic dodecahedron with vertices labeled in the homogeneous coordinates.

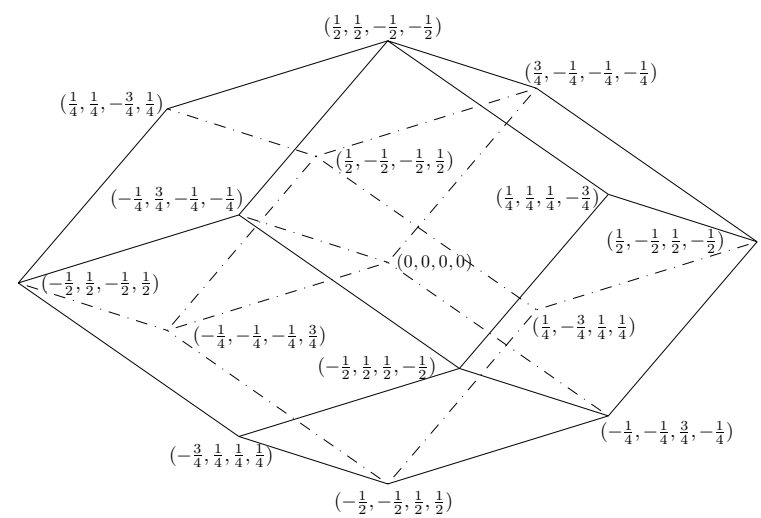

FiguRE 3.3. Rhombic dodecahedron labeled in homogeneous coordinates. 
Under the change of variables (3.2), the integral over $\Omega_{H}$ is given by

$$
\frac{1}{\left|\Omega_{H}\right|} \int_{\Omega_{H}} f(\mathbf{t}) d \mathbf{t}=\frac{1}{|\Omega|} \int_{\Omega} f(x) d x .
$$

By Fuglede's theorem, $\left\{e^{2 \pi i k^{\text {tr }} A^{-1} x}: k \in \mathbb{Z}^{3}\right\}$ forms an orthonormal basis in $L^{2}(\Omega)$. We would like to reformulate the exponential functions $e^{2 \pi i k^{\text {tr }} A^{-1} x}$ so that they are indexed by homogeneous coordinates. For this purpose, we denote by

$$
\mathbb{Z}_{H}^{4}:=\mathbb{Z}^{4} \cap \mathbb{R}_{H}^{4}=\left\{\mathbf{k} \in \mathbb{Z}^{4}: k_{1}+k_{2}+k_{3}+k_{4}=0\right\}
$$

the set of integers in homogeneous coordinates and introduce the notation

$$
\mathbb{H}:=\left\{\mathbf{k} \in \mathbb{Z}_{H}^{4}: k_{1} \equiv k_{2} \equiv k_{3} \equiv k_{4}(\bmod 4)\right\} .
$$

The definition of the matrix $H$ shows that if $k \in \mathbb{Z}^{3}$, then $H k \in \mathbb{Z}_{H}^{4}$. For $k \in \mathbb{Z}^{3}$, set $\mathbf{j}=4 H\left(A^{\operatorname{tr}} A\right)^{-1} k \in \mathbb{Z}_{H}^{4}$. A quick computation reveals that $\mathbf{j} \in \mathbb{H}$. Moreover, given $\mathbf{j} \in \mathbb{H}$, it follows from $A=U^{\mathrm{tr}} H$ that $k=\frac{1}{4} A^{\mathrm{tr}} U^{\mathrm{tr}} \mathbf{j}$, which is easily seen to be in $\mathbb{Z}^{3}$. Furthermore, we have

$$
k^{\text {tr }} A^{-1} x=\frac{1}{4} \mathbf{j}^{\operatorname{tr}} U A A^{-1} x=\frac{1}{4} \mathbf{j}^{\operatorname{tr}} U x=\frac{1}{4} \mathbf{j} \cdot \mathbf{t} .
$$

Consequently, we can index the exponentials by $\mathbf{j} \in \mathbb{H}$ and the exponent $2 \pi i k^{\text {tr }} A^{-1} x$ becomes $\frac{\pi i}{2} \mathbf{j} \cdot \mathbf{t}$. Let us introduce the notation

$$
\phi_{\mathbf{j}}(\mathbf{t}):=e^{\frac{\pi i}{2} \mathbf{j} \cdot \mathbf{t}}, \quad \mathbf{j} \in \mathbb{H} .
$$

Then, using (3.2) and recalling (2.2), the Fuglede Theorem becomes the following:

Proposition 3.1. For $\mathbf{k}, \mathbf{j} \in \mathbb{H}$,

$$
\left\langle\phi_{\mathbf{k}}, \phi_{\mathbf{j}}\right\rangle=\frac{1}{2} \int_{\Omega_{H}} \phi_{\mathbf{k}}(\mathbf{t}) \overline{\phi_{\mathbf{j}}(\mathbf{t})} d \mathbf{t}=\delta_{\mathbf{k}, \mathbf{j}} .
$$

Furthermore, $\left\{\phi_{\mathbf{j}}: \mathbf{j} \in \mathbb{H}\right\}$ is an orthonormal basis of $L^{2}\left(\Omega_{H}\right)$.

Given $f$ defined on $\Omega$, the mapping (3.2) shows that $f(x)=f\left(U^{\mathrm{tr}} \mathbf{t}\right)=g(\mathbf{t})$ is the function in homogeneous coordinates. Since $A=U^{\text {tr }} H$, a function $f$ being periodic with respect to the lattice $A \mathbb{Z}^{d}$ becomes, in homogeneous coordinates, the following definition:

Definition 3.2. A function $f$ is $H$-periodic if it is periodic with respect to the fcc lattice; that is, $f(\mathbf{t})=f(\mathbf{t}+H k)$ for $x \in \Omega_{H}$ and $k \in \mathbb{Z}^{3}$.

Using the explicit form of the matrix $H$, it is easy to see that the following holds:

Lemma 3.3. A function $f(\mathbf{t})$ is $H$-periodic if and only if

$$
f(\mathbf{t})=f(\mathbf{s}), \quad \mathbf{t}-\mathbf{s} \in \mathbb{Z}_{H}^{4},
$$

or equivalently,

$$
f\left(\mathbf{t}+k \mathbf{e}_{i, j}\right)=f(\mathbf{t}), \quad k \in \mathbb{Z}, \quad 1 \leq i<j \leq 4,
$$

where $\mathbf{e}_{i, j}:=e_{i}-e_{j}$ and $\left\{e_{1}, e_{1}, e_{3}, e_{4}\right\}$ is the standard basis of $\mathbb{R}^{4}$.

Evidently, the functions $\phi_{\mathbf{j}}(\mathbf{t})$ in (3.6) are $H$-periodic. Furthermore, Proposition 3.1 shows that an $H$-periodic function $f$ can be expanded into a Fourier series

$$
f \sim \sum_{\mathbf{k} \in \mathbb{H}} \widehat{f}_{\mathbf{k}} \phi_{\mathbf{k}}(\mathbf{t}), \quad \text { where } \quad \widehat{f}_{\mathbf{k}}:=\frac{1}{2} \int_{\Omega_{H}} f(\mathbf{t}) \phi_{-\mathbf{k}}(\mathbf{t}) d \mathbf{t} .
$$


3.2. Boundary of the rhombic dodecahedron. In order to carry out the discrete Fourier analysis on the rhombic dodecahedron, we need to have a detailed knowledge of the boundary of the polyhedral.

We use the standard set theory notations $\partial \Omega, \Omega^{\circ}$ and $\bar{\Omega}$ to denote the boundary, the interior and the closure of $\Omega$, respectively. Clearly, $\bar{\Omega}=\Omega^{\circ} \cup \partial \Omega$. A rhombic dodecahedron has 12 faces, 24 edges and 14 vertices. Since we will consider points on the boundary, we need to distinguish a face with its edges and without its edges, and an edge with its end points and without its end points. In the following, when we say a face or an edge, we mean the open set, that is, without its edges or end points, respectively.

We shall work with homogeneous coordinates. To describe the boundary of $\Omega_{H}$ we set $\mathbb{N}_{4}:=\{1,2,3,4\}$. For $i, j \in \mathbb{N}_{4}$ and $i \neq j$, define

$$
F_{i, j}=\left\{\mathbf{t} \in \bar{\Omega}_{H}: t_{i}-t_{j}=1\right\} .
$$

There are a total $2\left(\begin{array}{l}4 \\ 2\end{array}\right)=12$ distinct $F_{i, j}$ and it easy to see that each $F_{i, j}$ stands for one face, with its edges, of the rhombic dodecahedron.

For nonempty subsets $I, J$ of $\mathbb{N}_{4}$, define

$$
\Omega_{I, J}:=\bigcap_{i \in I, j \in J} F_{i, j}=\left\{\mathbf{t} \in \bar{\Omega}_{H}: t_{j}=t_{i}-1 \text {, for all } i \in I, j \in J\right\} .
$$

Lemma 3.4. Let $I, J, I_{i}, J_{i}$ be nonempty subsets of $\mathbb{N}_{4}$. Then

(i) $\Omega_{I, J}=\emptyset$ if and only if $I \cap J \neq \emptyset$.

(ii) $\Omega_{I_{1}, J_{1}} \cap \Omega_{I_{2}, J_{2}}=\Omega_{I, J}$ if $I_{1} \cup I_{2}=I$ and $J_{1} \cup J_{2}=J$.

Proof. It is obvious that $\Omega_{I, J} \neq \emptyset$ if $I \cap J=\emptyset$. On the other hand, if $I \cap J \neq \emptyset$ and $i=j \in I \cap J$, then $t_{i}-t_{j}=0 \neq 1$, which shows that $\Omega_{I, J}=\emptyset$. This proves (i).

If either $I_{1} \cap J_{1} \neq \emptyset$ or $I_{2} \cap J_{2} \neq \emptyset$, then $\Omega_{I_{1}, J_{1}} \cap \Omega_{I_{2}, J_{2}}=\Omega_{I, J}=\emptyset$ by (i). If $I_{1} \cap J_{1}=I_{2} \cap J_{2}=\emptyset$ and $i_{\nu} \in I_{\nu}, j_{\nu} \in J_{\nu}$ for $\nu=1,2$, then

$$
t_{i_{1}}-t_{j_{2}}=t_{i_{1}}-t_{i_{2}}+1 \leq 1 \text { and } \quad-1 \leq t_{j_{1}}-t_{i_{2}}=t_{i_{1}}-t_{i_{2}}-1
$$

which implies $t_{i_{1}}-t_{i_{2}}=0$ so that $t_{j_{1}}=t_{j_{2}}=t_{i_{1}}-1=t_{i_{2}}-1$ and proves $\Omega_{I_{1}, J_{1}} \cap \Omega_{I_{2}, J_{2}}=\Omega_{I, J}$.

Edges are intersections of faces and vertices are intersections of edges. Lemma 3.4 gives us information about the intersections. To make clear the structure of the boundary $\partial \Omega_{H}$, we introduce the notation

$$
\begin{aligned}
& \mathcal{K}=\left\{(I, J): I, J \subset \mathbb{N}_{4} ; I \cap J=\emptyset\right\}, \\
& \mathcal{K}_{0}=\{(I, J) \in \mathcal{K}: i<j, \text { for all }(i, j) \in(I, J)\} .
\end{aligned}
$$

Definition 3.5. For $(I, J) \in \mathcal{K}$, the boundary element $B_{I, J}$ of the dodecahedron,

$$
B_{I, J}:=\left\{\mathbf{t} \in \Omega_{I, J}: \mathbf{t} \notin \Omega_{I_{1}, J_{1}} \text { for all }\left(I_{1}, J_{1}\right) \in \mathcal{K} \text { with }|I|+|J|<\left|I_{1}\right|+\left|J_{1}\right|\right\},
$$

is called a face if $|I|+|J|=2$, an edge if $|I|+|J|=3$ and a vertex if $|I|+|J|=4$.

For the faces and the edges, the boundary elements represent the interiors. In fact, it is easy to see that $B_{\{i\},\{j\}}=F_{i, j}^{\circ}$ and, for example, $B_{\{i\},\{j, k\}}=\left(F_{i, j} \cap F_{i, k}\right)^{\circ}$ for distinct integers $i, j, k \in \mathbb{N}_{4}$. 
Furthermore, for $0<i, j<i+j \leq 4$, we define

$$
\begin{aligned}
& \mathcal{K}^{i, j}:=\{(I, J) \in \mathcal{K}:|I|=i,|J|=j\}, \quad B^{i, j}:=\bigcup_{(I, J) \in \mathcal{K}^{i, j}} B_{I, J} \\
& \mathcal{K}_{0}^{i, j}:=\left\{(I, J) \in \mathcal{K}_{0}:|I|=i,|J|=j\right\}, \quad B_{0}^{i, j}:=\bigcup_{(I, J) \in \mathcal{K}_{0}^{i, j}} B_{I, J} .
\end{aligned}
$$

Note that $B^{i, j}$ is the union of boundary points in those $B_{I, J}$ for which $|I|=i$ and $|J|=j$.

Proposition 3.6. Let $(I, J) \in \mathcal{K}$ and $\left(I_{1}, J_{1}\right) \in \mathcal{K}$.

(i) $B_{I, J} \cap B_{I_{1}, J_{1}}=\emptyset$, if $I \neq I_{1}$ and $J \neq J_{1}$.

(ii) $\bar{\Omega}_{H} \backslash \Omega_{H}^{\circ}=\bigcup_{(I, J) \in \mathcal{K}} B_{I, J}=\bigcup_{0<i, j<i+j \leq 4} B^{i, j}$.

(iii) $\Omega_{H} \backslash \Omega_{H}^{\circ}=\bigcup_{(I, J) \in \mathcal{K}_{0}} B_{I, J}=\bigcup_{0<i, j<i+j \leq 4} B_{0}^{i, j}$.

Proof. If $B_{I, J} \cap B_{I_{1}, J_{1}} \neq \emptyset$, then $|I|+|J|=\left|I_{1}\right|+\left|J_{1}\right|$. Moreover, if $\mathbf{t} \in B_{I, J} \cap B_{I_{1}, J_{1}}$, then $\mathbf{t} \in \Omega_{I, J} \cap \Omega_{I_{1}, J_{1}}=\Omega_{I \cup I_{1}, J \cup J_{1}}$ by Lemma 3.4 which implies that $|I|+|J| \geq$ $\left|I \cup I_{1}\right|+\left|J \cup J_{1}\right|$. Thus we must have $I=I_{1}=I \cup I_{1}$ and $J=J_{1}=J \cup J_{1}$, which contradicts the assumption and proves (i).

To prove (ii), we define for $\mathbf{t} \in \partial \Omega_{H}, I=\left\{i \in \mathbb{N}_{4}: \exists j \in \mathbb{N}_{4}\right.$ such that $\left.t_{i}-t_{j}=1\right\}$ and $J=\left\{j \in \mathbb{N}_{4}: \exists i \in \mathbb{N}_{4}\right.$ such that $\left.t_{i}-t_{j}=1\right\}$. Clearly, $(I, J) \in \mathcal{K}$ and $\mathbf{t} \in B_{I, J}$, which proves the first equal sign of (ii). The second equal sign follows from the definition of $B^{i, j}$. Since

$$
\Omega_{H} \backslash \Omega_{H}^{\circ}=\left\{\mathbf{t} \in \bar{\Omega}_{H} \backslash \Omega_{H}^{\circ}: t_{i}-t_{j}>-1, \forall i<j\right\},
$$

the part (iii) follows immediately from (ii).

The above proposition provides a decomposition of the boundary into nonoverlapping boundary elements. To make each boundary element explicit, we use symmetry. Let $\mathcal{G}=S_{4}$ be the permutation group of four elements. For $\mathbf{t} \in \mathbb{R}_{H}^{4}$ and $\sigma \in \mathcal{G}$, the action of $\sigma$ on $\mathbf{t}$ is denoted by $\mathbf{t} \sigma$, which means the permutation of the elements of $\mathbf{t}$ by $\sigma$. A moment of reflection shows that, for $(I, J) \subset \mathcal{K}$,

$$
B^{|I|,|J|}=\bigcup_{\sigma \in \mathcal{G}} B_{I, J} \sigma:=\left\{\mathbf{t} \sigma: \mathbf{t} \in B_{I, J}, \sigma \in \mathcal{G}\right\} .
$$

Later in the section we will need to consider points on the boundary elements that are congruent module $H$. For $(I, J) \subset \mathcal{K}$ we further define

$$
\left[B_{I, J}\right]:=\left\{B_{I, J}+\mathbf{k}: \mathbf{k} \in \mathbb{Z}_{H}^{4}\right\} \cap \bar{\Omega}_{H}=\left\{\mathbf{t}+\mathbf{k} \in \bar{\Omega}_{H}: \mathbf{t} \in B_{I, J}, \mathbf{k} \in \mathbb{Z}_{H}^{4}\right\} .
$$

Since $\left[B_{I, J}\right]$ is a subset of $\bar{\Omega}_{H}$ and $B_{I, J}$ is a boundary element, we see that $\left[B_{I, J}\right]$ consists of exactly those boundary elements that can be obtained from $B_{I, J}$ by congruent modulus $H$, as confirmed by the following lemma.

Lemma 3.7. If $\mathbf{t}, \mathbf{s} \in \Omega_{H}$ and $\mathbf{s} \equiv \mathbf{t}(\bmod H)$, then $\mathbf{t}=\mathbf{s}$.

Proof. By Lemma 3.3, if $\mathbf{t}, \mathbf{s} \in \Omega_{H}$ and $\mathbf{t} \equiv \mathbf{s}(\bmod H)$, then $\mathbf{s}-\mathbf{t} \in \mathbb{Z}_{H}^{4}$ and, set $\mathbf{k}:=\mathbf{s}-\mathbf{t},-1 \leq k_{i}-k_{j} \leq 1$ for all $i, j \in \mathbb{N}_{4}$. The last condition means that either $k_{i} \in\{0,1\}$ for all $i \in \mathbb{N}_{4}$ or $k_{i} \in\{0,-1\}$ for all $i \in \mathbb{N}_{4}$. The homogenous condition $k_{1}+k_{2}+k_{3}+k_{4}=0$ then shows that $k_{1}=k_{2}=k_{3}=k_{4}=0$ or $\mathbf{s}=\mathbf{t}$. 
As an example, we have

$$
\begin{aligned}
& B_{\{1\},\{2,3\}}=\left\{(t, t-1, t-1,2-3 t): \frac{1}{2}<t<\frac{3}{4}\right\}, \\
& B_{\{1,2\},\{3\}}=\left\{(1-t, 1-t,-t, 3 t-2): \frac{1}{2}<t<\frac{3}{4}\right\},
\end{aligned}
$$

and from the explicit description of $B_{\{1\},\{2,3\}}$ we deduce

$$
\begin{aligned}
{\left[B_{\{1\},\{2,3\}}\right] } & =B_{\{1\},\{2,3\}} \cup\left(B_{\{1\},\{2,3\}}+(-1,1,0,0)\right) \cup\left(B_{\{1\},\{2,3\}}+(-1,0,1,0)\right) \\
(3.10) & =B_{\{1\},\{2,3\}} \cup B_{\{2\},\{1,3\}} \cup B_{\{3\},\{1,2\}} .
\end{aligned}
$$

Others can be deduced similarly. The last equation indicates that $\left[B_{I, J}\right]$ is a union of $B_{I^{\prime}, J^{\prime}}$, which we make precise below.

Let $\sigma_{i j}$ denote the element in $\mathcal{G}$ that interchanges $i$ and $j$; then $\mathbf{t} \sigma_{i j}=\mathbf{t}-$ $\left(t_{i}-t_{j}\right) \mathbf{e}_{i, j}$. For a nonempty set $I \subset \mathbb{N}_{4}$, define $\mathcal{G}_{I}:=\left\{\sigma_{i j}: i, j \in I\right\}$, where we take $\sigma_{i j}=\sigma_{j i}$ and take $\sigma_{j j}$ as the identity element. It is easy to verify that $\mathcal{G}_{I}$ forms a subgroup of $\mathcal{G}=\mathcal{S}_{4}$ of order $|I|$.

Lemma 3.8. Let $(I, J) \in \mathcal{K}$. Then

$$
\left[B_{I, J}\right]=\bigcup_{\sigma \in \mathcal{G}_{I \cup J}} B_{I, J} \sigma .
$$

Proof. For any $i, j \in I \cup J$, the definition of $B_{I, J}$ shows that $\mathbf{t} \sigma_{i j}-\mathbf{t}=\left(t_{j}-t_{i}\right) \mathbf{e}_{i j} \in$ $\mathbb{Z}_{H}^{4}$ for all $\mathbf{t} \in B_{I, J}$. It then follows from $\mathbf{t} \sigma_{i j} \in \bar{\Omega}_{H}$ that $B_{I, J} \sigma_{i j} \subseteq\left[B_{I, J}\right]$. Consequently, $\bigcup_{\sigma \in \mathcal{G}_{I \cup J}} B_{I, J} \sigma \subseteq\left[B_{I, J}\right]$.

On the other hand, for any $\mathbf{s} \in\left[B_{I, J}\right]$ there exists $\mathbf{t} \in B_{I, J}$ such that $\mathbf{s}-\mathbf{t} \in \mathbb{Z}_{H}^{4}$. It follows from Proposition 3.6 and Lemma 3.7 that $\mathbf{s} \in B_{I_{1}, J_{1}}$ for a pair $\left(I_{1}, J_{1}\right) \in$ $\mathcal{K}$. By the definitions of $B_{I, J}$ and $\bar{\Omega}_{H}$, there exist $t, s \in \mathbb{R}$ such that

$$
\begin{aligned}
& -\frac{3}{4} \leq t-1=t_{j}<t_{l}<t_{i}=t \leq \frac{3}{4}, \quad i \in I, \quad j \in J, l \notin I \cup J, \\
& -\frac{3}{4} \leq s-1=s_{j}<s_{l}<s_{i}=s \leq \frac{3}{4}, \quad i \in I_{1}, \quad j \in J_{1}, l \notin I_{1} \cup J_{1} .
\end{aligned}
$$

Since $\mathbf{s}-\mathbf{t} \in \mathbb{Z}_{H}^{4}$, the above inequalities imply that $s_{i}-t_{i} \in\{-1,0,1\}$ for all $i \in \mathbb{N}_{4}$. We claim that $t=s$. Assume otherwise, say $s>t$. For $i \in I_{1}, s_{i}-t_{i} \geq s-t>0$ so that $s_{i}-t_{i}=1$; while for $i \notin I_{1}, s_{i}-t_{i} \geq s-1-t>-1$ so that $s_{i}-t_{i} \in\{1,0\}$. It then follows that $\sum_{i \in \mathbb{N}_{4}}\left(s_{i}-t_{i}\right)>0$, which poses a contradiction to the homogeneity of $\mathbf{s}-\mathbf{t}$. Hence we must have $s=t$. With $s=t$, it is then easy to see that $s_{i}-s=s_{i}-t_{i} \in\{0,-1\}$ for $i \in I, s_{j}-s+1=s_{j}-t_{j} \in\{0,1\}$ for $j \in J$, and $s-1<s_{l}=t_{l}<s$ for $l \notin I \cup J$. This shows that $I \cup J=I_{1} \cup J_{1}$ and $\sum_{i \in I \cup J} t_{i}=\sum_{i \in I_{1} \cup J_{1}} s_{i}$. Meanwhile, we note that $\sum_{i \in I \cup J} t_{i}=t(|I|+|J|)-|J|$ and $\sum_{i \in I_{1} \cup J_{1}} s_{i}=s\left(\left|I_{1}\right|+\left|J_{1}\right|\right)-\left|J_{1}\right|$. It then follows that $|J|=\left|J_{1}\right|$ and $|I|=\left|I_{1}\right|$. Consequently, $\mathbf{s}=\mathbf{t} \sigma$ for a $\sigma \in \mathcal{G}_{I \cup J}$ and $\left[B_{I, J}\right] \subseteq \bigcup_{\sigma \in \mathcal{G}_{I \cup J}} B_{I, J} \sigma$. This completes the proof of the lemma.

Since $\mathcal{K}^{i, j}$ can be obtained from $\mathcal{K}_{0}^{i, j}$ from the action of $\mathcal{G}$, it follows that

$$
B^{i, j}=\bigcup_{(I, J) \in \mathcal{K}_{0}^{i, j}}\left[B_{I, J}\right]=\bigcup_{B \in B_{0}^{i, j}}[B], \quad 0<i, j<i+j \leq 4 .
$$

We also note that $\left[B_{I, J}\right] \cap\left[B_{I_{1}, J_{1}}\right]=\emptyset$ if $(I, J) \neq\left(I_{1}, J_{1}\right)$ for $(I, J) \in \mathcal{K}_{0}$ and $\left(I_{1}, J_{1}\right) \in \mathcal{K}_{0}$, which shows that (3.12) is a nonoverlapping partition. 
If $|I|+|J|=3$ or $B_{I, J}$ is an edge, then we have

$$
\begin{aligned}
& B^{1,2}=\left[B_{\{1\},\{2,3\}}\right] \cup\left[B_{\{1\},\{2,4\}}\right] \cup\left[B_{\{1\},\{3,4\}}\right] \cup\left[B_{\{2\},\{3,4\}}\right], \\
& B^{2,1}=\left[B_{\{1,2\},\{3\}}\right] \cup\left[B_{\{1,2\},\{4\}}\right] \cup\left[B_{\{1,3\},\{4\}}\right] \cup\left[B_{\{2,3\},\{4\}}\right],
\end{aligned}
$$

where we recall that $B_{\{1\},\{2,3\}}$ and $B_{\{1,2\},\{3\}}$ are given in (3.9),

$$
\begin{aligned}
& B_{\{1\},\{2,4\}}=B_{\{1\},\{2,3\}} \sigma_{34}, \quad B_{\{1,2\},\{4\}}=B_{\{1,2\},\{3\}} \sigma_{34}, \\
& B_{\{1\},\{3,4\}}=B_{\{1\},\{2,3\}} \sigma_{24}, \quad B_{\{1,3\},\{4\}}=B_{\{1,2\},\{3\}} \sigma_{23} \sigma_{34}, \\
& B_{\{2\},\{3,4\}}=B_{\{1,2\},\{3\}} \sigma_{12} \sigma_{24}, \quad B_{\{2,3\},\{4\}}=B_{\{1,2\},\{3\}} \sigma_{13} \sigma_{34} .
\end{aligned}
$$

If $|I|+|J|=4$, then

$$
\begin{aligned}
B^{1,3} & =\left[\left\{\left(\frac{1}{4}, \frac{1}{4}, \frac{1}{4},-\frac{3}{4}\right)\right\}\right], \quad B^{2,2}=\left[\left\{\left(\frac{1}{2}, \frac{1}{2},-\frac{1}{2},-\frac{1}{2}\right\}\right)\right] \\
B^{3,1} & =\left[\left\{\left(\frac{3}{4},-\frac{1}{4},-\frac{1}{4},-\frac{1}{4}\right)\right\}\right] .
\end{aligned}
$$

3.3. Dodecahedral Fourier partial sum. In order to apply the general result on discrete Fourier analysis in the previous section to fcc lattice, we choose $A=A$ and $B=n A$ with $n$ being a positive integer. Then the matrix

$$
N=B^{\operatorname{tr}} A=\left(\begin{array}{ccc}
2 n & n & n \\
n & 2 n & n \\
n & n & 2 n
\end{array}\right)
$$

has integer entries. Note that $N$ is now a symmetric matrix so that $\Lambda_{N}=\Lambda_{N^{\mathrm{tr}}}$, and it is easy to see that $\Lambda_{N}^{\dagger}=\Lambda_{N}$. Recall the definition of $\mathbb{H}$ in (3.5). Using again $\mathbf{j}=4 H\left(A^{\text {tr }} A\right)^{-1} k \in \mathbb{Z}_{H}^{4}$, it is easy to see that $k \in \Lambda_{N}$ becomes $\mathbf{j} \in \mathbb{H}_{n}$, where

$$
\mathbb{H}_{n}:=\left\{\mathbf{k} \in \mathbb{H}: \frac{\mathbf{k}}{4 n} \in \Omega_{H}\right\}=\left\{\mathbf{k} \in \mathbb{H}:-4 n<k_{i}-k_{j} \leq 4 n, 1 \leq i<j \leq 4\right\} .
$$

The finite dimensional space $\mathcal{H}_{N}$ of exponentials in Theorem 2.5 becomes

$$
\mathcal{H}_{n}:=\operatorname{span}\left\{\phi_{\mathbf{k}}: \mathbf{k} \in \mathbb{H}_{n}\right\} \quad \text { with } \operatorname{dim} \mathcal{H}_{n}=\operatorname{det}(N)=4 n^{3} .
$$

Note that the points in $\mathbb{H}_{n}$ are not symmetric under $\mathcal{G}$, since points on half of the boundary are not included. For reasons of symmetry, we further define

$$
\mathbb{H}_{n}^{*}:=\left\{\mathbf{k} \in \mathbb{H}: \frac{\mathbf{k}}{4 n} \in \bar{\Omega}_{H}\right\}=\left\{\mathbf{k} \in \mathbb{H}:-4 n \leq k_{i}-k_{j} \leq 4 n, 1 \leq i<j \leq 4\right\} .
$$

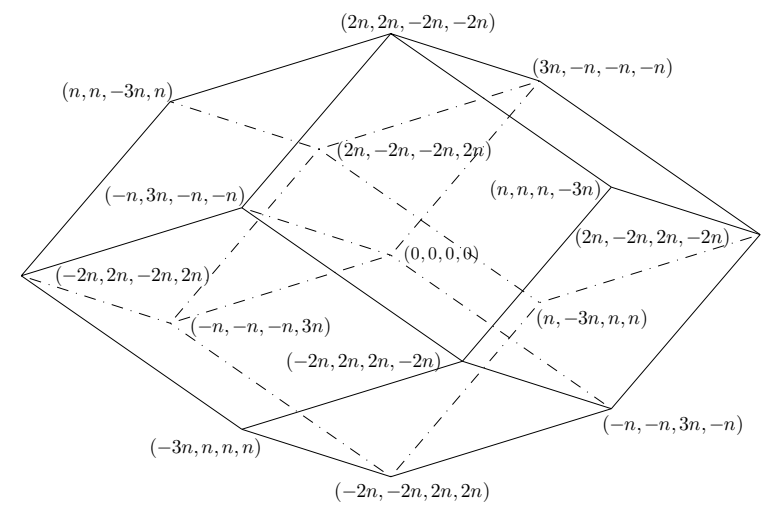

FiguRE 3.4. $\mathbb{H}_{n}^{*}=\left\{\mathbf{t} \in \mathbb{H}:-4 n \leq t_{i}-t_{j} \leq 4 n\right\}$. 
For the Fourier expansion (3.7) of an $\mathrm{H}$-periodic function, we define its dodecahedral partial sum as

$$
S_{n} f(\mathbf{t}):=\sum_{\mathbf{k} \in \mathbb{H}_{n}^{*}}\left\langle f, \phi_{\mathbf{k}}\right\rangle \phi_{\mathbf{k}}(\mathbf{t})=\frac{1}{2} \int_{\Omega_{H}} f(\mathbf{s}) D_{n}^{H}(\mathbf{t}-\mathbf{s}) d \mathbf{s},
$$

where $D_{n}^{H}$ is the Dirichlet kernel for the dodecahedral partial sum

$$
D_{n}^{H}(\mathbf{t}):=\sum_{\mathbf{k} \in \mathbb{H}_{n}^{*}} e^{\frac{\pi i}{2} \mathbf{k} \cdot \mathbf{t}} .
$$

Our immediate goal is to find a compact formula for the Dirichlet kernel $D_{n}^{H}$. We start with an observation that the index set $\mathbb{H}_{n}^{*}$ can be partitioned into four congruent parts, each within a parallelepiped, as shown in Figures 3.5-3.8.

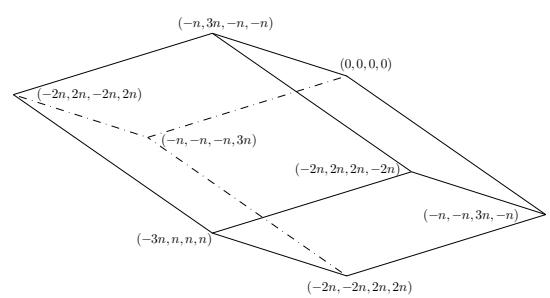

FIGURE 3.5. $\mathbb{H}_{n}^{(1)}$

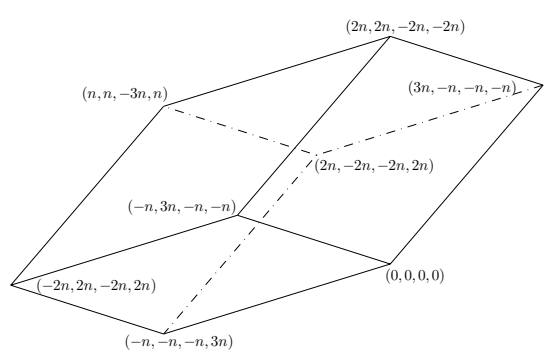

FIGURE 3.7. $\mathbb{H}_{n}^{(3)}$

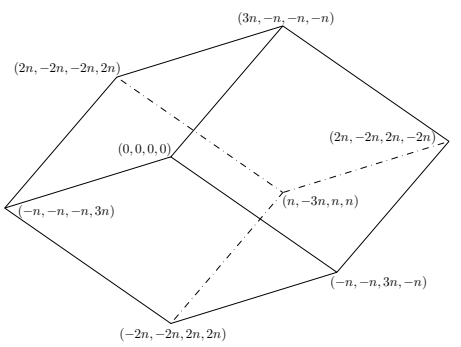

FIGURE 3.6. $\mathbb{H}_{n}^{(2)}$

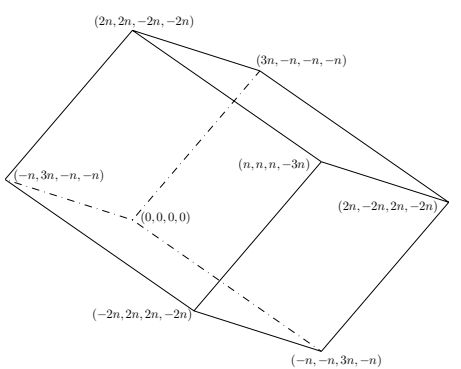

FIGURE 3.8. $\mathbb{H}_{n}^{(4)}$

Lemma 3.9. Define $\mathbb{H}_{n}^{(k)}:=\left\{\mathbf{j} \in \mathbb{H}: 0 \leq j_{l}-j_{k} \leq 4 n, l \in \mathbb{N}_{4}\right\}$ for $k \in \mathbb{N}_{4}$ and

$$
\mathbb{H}_{n}^{J}:=\left\{\mathbf{k} \in \mathbb{H}: k_{i}=k_{j}, \forall i, j \in J, \text { and } 0 \leq k_{i}-k_{j} \leq 4 n, \forall j \in J, \forall i \in \mathbb{N}_{4} \backslash J\right\}
$$

for $\emptyset \subset J \subseteq \mathbb{N}_{4}$. Then

$$
\mathbb{H}_{n}^{*}=\bigcup_{j \in \mathbb{N}_{4}} \mathbb{H}_{n}^{(j)} \quad \text { and } \quad \mathbb{H}_{n}^{J}=\bigcap_{j \in J} \mathbb{H}_{n}^{(j)}
$$

Proof. For $\mathbf{k} \in \mathbb{H}_{n}^{*}$, let $k_{i}=\min \left\{k_{1}, k_{2}, k_{3}, k_{4}\right\}$. Then $\mathbf{k} \in \mathbb{H}_{n}^{(i)}$, which implies that $\mathbb{H}_{n}^{*} \subseteq \bigcup_{i \in \mathbb{N}_{4}} \mathbb{H}_{n}^{(i)}$. Since $\mathbb{H}_{n}^{(i)} \subset \mathbb{H}_{n}^{*}$ for each $i \in \mathbb{N}_{4}$, it follows that $\mathbb{H}_{n}^{*}=\bigcup_{i \in \mathbb{N}_{4}} \mathbb{H}_{n}^{(i)}$. 
If $\mathbf{k} \in \mathbb{H}_{n}^{(i)} \cap \mathbb{H}_{n}^{(j)}$, then $0 \leq k_{i}-k_{j} \leq 0$; that is, $k_{i}=k_{j}$. It follows that if $\mathbf{k} \in \bigcap_{j \in J} \mathbb{H}_{n}^{(j)}$, then $k_{i}=k_{j}, \forall i, j \in J$, which implies $\bigcap_{j \in J} \mathbb{H}_{n}^{(j)} \subseteq \mathbb{H}_{n}^{J}$. Since $\mathbb{H}_{n}^{J} \subset \mathbb{H}_{n}^{(j)}$ by definition, we conclude that $\mathbb{H}_{n}^{J}=\bigcap_{j \in J} \mathbb{H}_{n}^{(j)}$.

Theorem 3.10. For $n \geq 0$,

$$
D_{n}^{H}(\mathbf{t})=\Theta_{n+1}(\mathbf{t})-\Theta_{n}(\mathbf{t}), \quad \text { where } \quad \Theta_{n}(\mathbf{t}):=\prod_{j=1}^{4} \frac{\sin \pi n t_{j}}{\sin \pi t_{j}} .
$$

Proof. Using the inclusion-exclusion relation of subsets, we have

$$
D_{n}^{H}(\mathbf{t})=\sum_{\emptyset \subset J \subseteq \mathbb{N}_{4}}(-1)^{|J|+1} \sum_{\mathbf{k} \in \mathbb{H}_{n}^{J}} e^{\frac{\pi i}{2} \mathbf{k} \cdot \mathbf{t}} .
$$

Fix $j \in J$, using the fact that $t_{j}=-\sum_{i \neq j} t_{i}$, we have

$$
\sum_{\mathbf{k} \in \mathbb{H}_{n}^{J}} e^{\frac{\pi i}{2} \mathbf{k} \cdot \mathbf{t}}=\sum_{\mathbf{k} \in \mathbb{H}_{n}^{J}} e^{\frac{\pi i}{2} \sum_{l \in \mathbb{N}_{4} \backslash J}\left(k_{l}-k_{j}\right) t_{l}}=\sum_{\mathbf{k} \in \mathbb{H}_{n}^{J}} \prod_{l \in \mathbb{N}_{4} \backslash J} e^{\frac{\pi i}{2}\left(k_{l}-k_{j}\right) t_{l}} .
$$

By the definition of $\mathbb{H}_{n}^{J}$ and the fact that $\mathbf{k} \in \mathbb{H}$ implies $k_{i} \equiv k_{j}(\bmod 4)$, we obtain

$$
\begin{aligned}
\sum_{\mathbf{k} \in \mathbb{H}_{n}^{J}} e^{\frac{\pi i}{2} \mathbf{k} \cdot \mathbf{t}} & =\prod_{l \in \mathbb{N}_{4} \backslash J J} \sum_{\substack{0 \leq k_{l}-k_{j} \leq 4 n \\
k_{l} \equiv k_{j}(\bmod 4)}} e^{\frac{\pi i}{2}\left(k_{l}-k_{j}\right) t_{l}} \\
& =\prod_{l \in \mathbb{N}_{4} \backslash J} \sum_{0 \leq k_{l} \leq n} e^{2 \pi i k_{l} t_{l}}:=\prod_{l \in \mathbb{N}_{4} \backslash J} K_{n}\left(t_{l}\right) .
\end{aligned}
$$

Consequently, we obtain

$$
D_{n}^{H}(\mathbf{t})=\sum_{\emptyset \subset J \subseteq \mathbb{N}_{4}}(-1)^{|J|+1} \prod_{l \in \mathbb{N}_{4} \backslash J} K_{n}\left(t_{l}\right)=\prod_{j \in \mathbb{N}_{4}} K_{n}\left(t_{j}\right)-\prod_{j \in \mathbb{N}_{4}}\left(K_{n}\left(t_{j}\right)-1\right)
$$

where the second equality is easily verified upon expanding the right-hand side explicitly. Thus, we conclude that

$$
\begin{aligned}
D_{n}^{H}(\mathbf{t}) & =\prod_{j=1}^{4} \frac{\mathrm{e}^{2 \pi i(n+1) t_{j}}-1}{e^{2 \pi i t_{j}}-1}-\prod_{j=1}^{4} \frac{\mathrm{e}^{2 \pi i(n+1) t_{j}}-\mathrm{e}^{2 \pi i t_{j}}}{e^{2 \pi i t_{j}}-1} \\
& =\prod_{j=1}^{4} \frac{e^{\pi i(n+1) t_{j}}-e^{-\pi i(n+1) t_{j}}}{e^{\pi i t_{j}}-e^{-\pi i t_{j}}} e^{\pi i n t_{j}}-\prod_{j=1}^{4} \frac{e^{\pi i n t_{j}}-e^{-\pi i n t_{j}}}{e^{\pi i t_{j}}-e^{-\pi i t_{j}}} e^{\pi i(n+1) t_{j}} \\
& =\prod_{j=1}^{4} \frac{\sin \pi(n+1) t_{j}}{\sin \pi t_{j}}-\prod_{j=1}^{4} \frac{\sin \pi n t_{j}}{\sin \pi t_{j}},
\end{aligned}
$$

where in the last step we have used the fact that $\prod_{j=1}^{4} e^{i \alpha t_{j}}=1$, which follows from the fact that $t_{1}+t_{2}+t_{3}+t_{4}=0$. This completes the proof.

As an immediate consequence of Theorem 3.10, we conclude that

$$
\left|\mathbb{H}_{n}^{*}\right|=D_{n}^{H}(0)=(n+1)^{4}-n^{4} .
$$

The explicit formula of the Dirichlet kernel also allows us to derive an estimate for the norm of the partial sum $S_{n} f$ in (3.16). Let $\|f\|_{\infty}$ denote the uniform of $f \in C\left(\bar{\Omega}_{H}\right)$ and let $\left\|S_{n}\right\|_{\infty}$ denote the operator norm of $S_{n}: C\left(\bar{\Omega}_{H}\right) \mapsto C\left(\bar{\Omega}_{H}\right)$. 
Theorem 3.11. There is a constant $c$ independent of $f$ and $n$ such that

$$
\left\|S_{n}\right\|_{\infty} \leq c(\log n)^{3} .
$$

Proof. From (3.16), a standard argument shows that the norm is given by

$$
\left\|S_{n}\right\|_{\infty}=\frac{1}{2} \max _{\mathbf{t} \in \bar{\Omega}_{H}} \int_{\Omega_{H}}\left|D_{n}^{H}(\mathbf{t}-\mathbf{s})\right| d \mathbf{s} .
$$

To estimate the integral, we use the first equation of (3.19) and the fact that

$$
K_{n}(t)=\sum_{j=0}^{n} e^{2 \pi i j t}=e^{\pi i n t} \frac{\sin \pi(n+1) t}{\sin \pi t},
$$

which leads to

$$
\begin{aligned}
\left\|S_{n}\right\|_{\infty} & \leq \frac{1}{2} \max _{\mathbf{t} \in \bar{\Omega}_{H}} \sum_{\emptyset \subset J \subseteq \mathbb{N}_{4}} \int_{\Omega_{H}} \prod_{l \in \mathbb{N}_{4} \backslash J}\left|K_{n}\left(t_{l}-s_{l}\right)\right| d \mathbf{s} \\
& \leq \frac{1}{2} \max _{\mathbf{t} \in \bar{\Omega}_{H}} \sum_{\emptyset \subset J \subseteq \mathbb{N}_{4}} \int_{\Omega_{H}} \prod_{l \in \mathbb{N}_{4} \backslash J}\left|\frac{\sin \pi(n+1)\left(t_{l}-s_{l}\right)}{\sin \pi\left(t_{l}-s_{l}\right)}\right| d \mathbf{s} .
\end{aligned}
$$

Since $J \neq \emptyset$, the above product contains at most three terms, and those that contain product of three terms dominate other integrals. Consequently, enlarging the domains of the integration and then using the periodicity of the trigonometric function, we conclude that

$$
\begin{aligned}
\left\|S_{n}\right\|_{\infty} & \leq c \int_{[-1,1]^{3}} \prod_{j=1}^{3}\left|\frac{\sin \pi(n+1) u_{j}}{\sin \pi u_{j}}\right| d u \\
& =c \prod_{j=1}^{3} \int_{-1}^{1}\left|\frac{\sin \pi(n+1) u_{j}}{\sin \pi u_{j}}\right| d u_{j} \leq c(\log n)^{3},
\end{aligned}
$$

where the last step follows from the usual estimate of the integral involved.

We expect that the estimate is sharp, that is, $\left\|S_{n}\right\|_{\infty} \geq c(\log n)^{3}$. To prove such a result would require a lower bound estimate of the integral of $\left|D_{n}^{H}(\mathbf{t}-\mathbf{s})\right|$ at one point in $\Omega_{H}$, likely at $\mathbf{s}=0$. However, this does not appear to be an easy task as there is a sum of four terms of the same type.

3.4. Discrete Fourier analysis on the rhombic dodecahedron. Using the set-up in the previous subsection, Theorem 2.5 in the homogeneous coordinates becomes the following proposition.

Proposition 3.12. For $n \geq 0$, define

$$
\langle f, g\rangle_{n}:=\frac{1}{4 n^{3}} \sum_{j \in \mathbb{H}_{n}} f\left(\frac{\mathbf{j}}{4 n}\right) \overline{g\left(\frac{\mathbf{j}}{4 n}\right)}, \quad f, g \in C\left(\bar{\Omega}_{H}\right) .
$$

Then

$$
\langle f, g\rangle=\langle f, g\rangle_{n}, \quad f, g \in \mathcal{H}_{n} .
$$

The point set $\mathbb{H}_{n}$, hence the inner product $\langle\cdot, \cdot\rangle_{n}$, is not symmetric on $\Omega_{H}$ in the sense that it contains only part of the points on the boundary. Using the periodicity, 
however, we can show that the inner product $\langle\cdot, \cdot\rangle_{n}$ is equivalent to a symmetric discrete inner product based on $\mathbb{H}_{n}^{*}$. To proceed, define

$$
\mathbb{H}_{n}^{\circ}:=\left\{\mathbf{j} \in \mathbb{H}: \frac{\mathbf{j}}{4 n} \in \Omega_{H}^{\circ}\right\}
$$

and, recall (3.8), for $0<i, j<i+j \leq 4$ define

$$
\mathbb{H}_{n}^{i, j}:=\left\{\mathbf{k} \in \mathbb{H}: \frac{\mathbf{k}}{4 n} \in B^{i, j}\right\}, \quad \mathbb{H}_{n, 0}^{i, j}:=\left\{\mathbf{k} \in \mathbb{H}: \frac{\mathbf{k}}{4 n} \in B_{0}^{i, j}\right\} .
$$

Recall that $B^{i, j}$ is a boundary element of $\Omega_{H}$, so that $\mathbb{H}_{n}^{i, j}$ describes those points $\mathbf{j}$ in $\mathbb{H}_{n}$ such that $\frac{\mathbf{j}}{4 n}$ are in $B^{i, j}$ of $\partial \Omega_{H}$. Furthermore, $\mathbb{H}_{n}^{i, j}=\left\{\mathbb{H}_{n, 0}^{i, j} \sigma: \sigma \in \mathcal{G}\right\}$. Using Proposition 3.6, it is easy to see that $\mathbb{H}_{n}^{i, j} \cap \mathbb{H}_{n}^{k, l}=\emptyset$ if $i \neq k, j \neq l$,

$$
\bigcup_{0<i, j<i+j \leq 4} \mathbb{H}_{n}^{i, j}=\mathbb{H}_{n}^{*} \backslash \mathbb{H}_{n}^{\circ} \quad \text { and } \bigcup_{0<i, j<i+j \leq 4} \mathbb{H}_{n, 0}^{i, j}=\mathbb{H}_{n} \backslash \mathbb{H}_{n}^{\circ}
$$

Lemma 3.13. For $n \geq 1,0<i, j<i+j \leq 4$,

$$
\left|\mathbb{H}_{n}^{\circ}\right|=n^{4}-(n-1)^{4}, \quad\left|\mathbb{H}_{n}^{i, j}\right|=\frac{4 !}{i ! j !(4-i-j) !}(n-1)^{4-i-j} .
$$

Proof. The first equation follows from $\left|\mathbb{H}_{n}^{\circ}\right|=\left|\mathbb{H}_{n-1}^{*}\right|=n^{4}-(n-1)^{4}$. The description of $B^{i, j}$ in Subsection 3.2 shows that $B^{i, j}$ has $\frac{4 !}{i ! j !(4-i-j) !}$ segments and each has $(n-1)^{4-i-j}$ points, which proves the second equation.

Definition 3.14. For $n \geq 0$ define the symmetric discrete inner product

$$
\langle f, g\rangle_{n}^{*}:=\frac{1}{4 n^{3}} \sum_{\mathbf{j} \in \mathbb{H}_{n}^{*}} c_{\mathbf{j}}^{(n)} f\left(\frac{\mathbf{j}}{4 n}\right) \overline{g\left(\frac{\mathbf{j}}{4 n}\right)}, \quad f, g \in C\left(\bar{\Omega}_{H}\right),
$$

where $c_{\mathbf{j}}^{(n)}=1$ if $\mathbf{j} \in \mathbb{H}_{n}^{\circ}$, and $c_{\mathbf{j}}^{(n)}=\frac{1}{\left(\begin{array}{c}i+j \\ i\end{array}\right)}$ if $\mathbf{j} \in \mathbb{H}_{n}^{i, j} ;$ more explicitly,

$$
c_{\mathbf{j}}^{(n)}= \begin{cases}1, \quad \mathbf{j} \in \mathbb{H}_{n}^{\circ}, & \left(n^{4}-(n-1)^{4} \text { points in the interior }\right), \\ \frac{1}{2}, \quad \mathbf{j} \in \mathbb{H}_{n}^{1,1}, & \left(12(n-1)^{2} \text { points on the faces }\right), \\ \frac{1}{3}, \quad \mathbf{j} \in \mathbb{H}_{n}^{1,2} \cup \mathbb{H}_{n}^{2,1}, & (2 \times 12(n-1) \text { points on the edges }), \\ \frac{1}{4}, \quad \mathbf{j} \in \mathbb{H}_{n}^{1,3} \cup \mathbb{H}_{n}^{3,1}, & (2 \times 4 \text { points on the vertices }), \\ \frac{1}{6}, \quad \mathbf{j} \in \mathbb{H}_{n}^{2,2}, & (6 \text { points on the vertices }) .\end{cases}
$$

It is easy to verify that $\sum_{\mathbf{j} \in \mathbb{H}_{n}^{*}} c_{\mathbf{j}}^{(n)}=4 n^{3}$, so that $\langle 1,1\rangle_{n}^{*}=1$. We prove the following result.

Theorem 3.15. For $n \geq 0$,

$$
\langle f, g\rangle=\langle f, g\rangle_{n}=\langle f, g\rangle_{n}^{*}, \quad f, g \in \mathcal{H}_{n} .
$$

Proof. For each $\mathbf{j} \in \mathbb{H}_{n, 0}^{i, j}$, we define

$$
\mathcal{S}_{\mathbf{j}}=\left\{\mathbf{k} \in \mathbb{H}_{n}^{*}: \frac{\mathbf{k}}{4 n} \equiv \frac{\mathbf{j}}{4 n} \quad(\bmod H)\right\} .
$$

It follows immediately from (3.12) that $\mathbb{H}_{n}^{i, j}=\bigcup_{\mathbf{k} \in \mathbb{H}_{n, 0}^{i, j}} \mathcal{S}_{\mathbf{k}}$. By (3.11), $\left[B^{I, J}\right]$ is the union of $\left(\begin{array}{c}|I|+|J| \\ |I|\end{array}\right)$ components of $B_{I, J} \sigma$. Consequently, it follows that $\left|\mathcal{S}_{\mathbf{j}}\right|=\left(\begin{array}{c}k+l \\ l\end{array}\right)$ 
for $\mathbf{j} \in \mathbb{H}_{n, 0}^{k, l}$. Let $f$ be an $H$-periodic function. Then

$$
\begin{aligned}
\sum_{\mathbf{j} \in \mathbb{H}_{n}^{*} \backslash \mathbb{H}_{n}^{\circ}} c_{\mathbf{j}}^{(n)} f\left(\frac{\mathbf{j}}{4 n}\right) & =\sum_{0<i, k<i+k \leq 4} \frac{1}{\left(\begin{array}{c}
i+k \\
i
\end{array}\right)} \sum_{\mathbf{j} \in \mathbb{H}_{n}^{i, k}} f\left(\frac{\mathbf{j}}{4 n}\right) \\
& =\sum_{0<i, k<i+k \leq 4} \frac{1}{\left(\begin{array}{c}
i+k \\
i
\end{array}\right)} \sum_{\mathbf{j} \in \mathbb{H}_{n, 0}^{i, k}} \sum_{\mathbf{k} \in \mathcal{S}_{\mathbf{j}}} f\left(\frac{\mathbf{k}}{4 n}\right) .
\end{aligned}
$$

Since $\left|\mathcal{S}_{\mathbf{j}}\right|=\left(\begin{array}{c}i+k \\ i\end{array}\right)$ for $\mathbf{j} \in \mathbb{H}_{n, 0}^{i, k}$, using the invariance of $f$, we then conclude that

$$
\sum_{\mathbf{j} \in \mathbb{H}_{n}^{*} \backslash \mathbb{H}_{n}^{\circ}} c_{\mathbf{j}}^{(n)} f\left(\frac{\mathbf{j}}{4 n}\right)=\sum_{0<i, k<i+k \leq 4} \frac{1}{\left(\begin{array}{c}
i+k \\
i
\end{array}\right)} \sum_{\mathbf{j} \in \mathbb{H}_{n, 0}^{i, k}}\left(\begin{array}{c}
i+k \\
i
\end{array}\right) f\left(\frac{\mathbf{j}}{4 n}\right)=\sum_{\mathbf{j} \in \mathbb{H}_{n} \backslash \mathbb{H}_{n}^{\circ}} f\left(\frac{\mathbf{j}}{4 n}\right) .
$$

Since $c_{\mathbf{j}}^{(n)}=1$ if $\mathbf{j} \in \mathbb{H}_{n}^{\circ}$, the proof is completed.

The discrete inner product is closely related to cubature formula, since Theorem 3.15 shows that the integral of $f \in \mathcal{H}_{n}$ agrees with the discrete sum over $\mathbb{H}_{n}^{*}$. In fact, more is true. Let us define by $\mathcal{T}_{n}$ the space of generalized trigonometric polynomials,

$$
\mathcal{T}_{n}:=\operatorname{span}\left\{\phi_{\mathbf{k}}: \mathbf{k} \in \mathbb{H}_{n}^{*}\right\} .
$$

Theorem 3.16. For $n \geq 0$, the cubature formula

$$
\frac{1}{2} \int_{\Omega} f(\mathbf{t}) d \mathbf{t}=\frac{1}{4 n^{3}} \sum_{\mathbf{j} \in \mathbb{H}_{n}^{*}} c_{\mathbf{j}}^{(n)} f\left(\frac{\mathbf{j}}{4 n}\right)
$$

is exact for all $f \in \mathcal{T}_{2 n-1}$.

Proof. If $\mathbf{k}, \mathbf{j} \in \mathbb{H}_{n}$, then the definition of $\mathbb{H}_{m}^{*}$ implies immediately that $\mathbf{k}-\mathbf{j} \in$ $\mathbb{H}_{2 n-1}^{*}$. Suppose now $\mathbf{j} \in \mathbb{H}_{2 n-1}^{*}$ and we may assume that $j_{1} \geq j_{2} \geq j_{3} \geq j_{4}$. There exists $\mathbf{k} \in \mathbb{H}_{n}^{*}$ such that $\mathbf{k}-\mathbf{j} \in \mathbb{H}_{n}^{*}$. Indeed, if $j_{1}-j_{3} \leq 4 n-4$, we can take $\mathbf{k}=(n, n, n,-3 n)$. If $j_{2}-j_{4} \leq 4 n-4$, we can take $\mathbf{k}=(3 n,-n,-n,-n)$. Finally, if both $j_{1}-j_{3} \geq 4 n$ and $j_{2}-j_{4} \geq 4 n$, then it follows from the definition of $\mathbb{H}_{2 n-1}^{*}$ that $j_{1}-j_{2} \leq 4 n-4$ and $j_{3}-j_{4} \leq 4 n-4$. In this case we can take $\mathbf{k}=(2 n, 2 n,-2 n,-2 n)$. Consequently, this shows that

$$
\mathbb{H}_{2 n-1}^{*}=\left\{\mathbf{l}: \mathbf{l}=\mathbf{k}-\mathbf{j}, \mathbf{k}, \mathbf{j} \in \mathbb{H}_{n}\right\} .
$$

Thus if $\phi_{\mathbf{j}} \in \mathcal{H}_{2 n-1}$, then $\mathbf{j} \in \mathbb{H}_{2 n-1}^{*}$ and there exist $\mathbf{k}, \mathbf{l} \in \mathbb{H}_{n}$ such that $\phi_{\mathbf{j}}=\phi_{\mathbf{k}} \overline{\phi_{\mathbf{l}}}$. Consequently, the stated result follows from Theorem 3.15 .

3.5. Interpolation on the rhombic dodecahedron. For the rhombic dodecahedron, Theorem 2.6 on interpolation becomes the following:

Proposition 3.17. For $n>0$, define

$$
\mathcal{I}_{n} f(\mathbf{t}):=\sum_{\mathbf{j} \in \mathbb{H}_{n}} f\left(\frac{\mathbf{j}}{4 n}\right) \Phi_{n}\left(\mathbf{t}-\frac{\mathbf{j}}{4 n}\right), \quad \text { where } \quad \Phi_{n}(\mathbf{t})=\frac{1}{4 n^{3}} \sum_{\mathbf{k} \in \mathbb{H}_{n}} \phi_{k}(\mathbf{t}),
$$

for $f \in C\left(\bar{\Omega}_{H}\right)$. Then $\mathcal{I}_{n} f \in \mathcal{H}_{n}$ and

$$
\mathcal{I}_{n} f\left(\frac{\mathbf{j}}{4 n}\right)=f\left(\frac{\mathbf{j}}{4 n}\right), \quad \forall \mathbf{j} \in \mathbb{H}_{n} .
$$


Again there is a lack of symmetry in the sense that $\mathcal{I}_{n}$ uses only points in $\mathbb{H}_{n}$, which contains only about half of the boundary points. We are more interested in another interpolation operator given below, defined over all points in $\mathbb{H}_{n}^{*}$. Although it does not interpolate at all points in $\mathbb{H}_{n}^{*}$, its symmetric form can be used to derive results on the tetrahedron in the next section. Recall $\mathcal{S}_{\mathbf{k}}$ defined in (3.22).

Theorem 3.18. For $n \geq 0$ and $f \in C\left(\bar{\Omega}_{H}\right)$, define

$$
\mathcal{I}_{n}^{*} f(\mathbf{t}):=\sum_{\mathbf{j} \in \mathbb{H}_{n}^{*}} f\left(\frac{\mathbf{j}}{4 n}\right) \ell_{\mathbf{j}, n}(\mathbf{t})
$$

where

$$
\ell_{\mathbf{j}, n}(\mathbf{t})=\Phi_{n}^{*}\left(\mathbf{t}-\frac{\mathbf{j}}{4 n}\right) \quad \text { and } \quad \Phi_{n}^{*}(\mathbf{t})=\frac{1}{4 n^{3}} \sum_{\mathbf{k} \in \mathbb{H}_{n}^{*}} c_{\mathbf{k}}^{(n)} \phi_{\mathbf{k}}(\mathbf{t}) .
$$

Then $\mathcal{I}_{n}^{*} f \in \mathcal{T}_{n}$ and it satisfies

$$
\mathcal{I}_{n}^{*} f\left(\frac{\mathbf{j}}{4 n}\right)= \begin{cases}f\left(\frac{\mathbf{j}}{4 n}\right), & \mathbf{j} \in \mathbb{H}_{n}^{\circ}, \\ \sum_{\mathbf{k} \in S_{\mathbf{j}}} f\left(\frac{\mathbf{k}}{4 n}\right), & \mathbf{j} \in \mathbb{H}_{n}^{*} \backslash \mathbb{H}_{n}^{\circ} .\end{cases}
$$

Furthermore, $\Phi_{n}^{*}(\mathbf{t})$ is a real function and it satisfies

$$
\begin{aligned}
& \Phi_{n}^{*}(\mathbf{t})=\frac{1}{4 n^{3}}\left[\frac{1}{2}\left(D_{n}^{H}(\mathbf{t})+D_{n-1}^{H}(\mathbf{t})\right)-\frac{1}{3} \sum_{\nu=1}^{4} \frac{\sin (n-1) \pi t_{\nu}}{\sin \pi t_{\nu}} \sum_{\substack{j=1 \\
j \neq \nu}}^{4} \cos n \pi\left(2 t_{j}+t_{\nu}\right)\right. \\
& \left.-\frac{1}{2} \sum_{j=1}^{4} \cos 2 \pi n t_{j}-\frac{1}{3} \sum_{1 \leq \mu<\nu \leq 4} \cos 2 \pi n\left(t_{\mu}+t_{\nu}\right)\right]
\end{aligned}
$$

Proof. By definition,

$$
\ell_{\mathbf{j}, n}\left(\frac{\mathbf{k}}{4 n}\right)=\Phi_{n}^{*}\left(\frac{\mathbf{k}-\mathbf{j}}{4 n}\right)=\frac{1}{4 n^{3}} \sum_{\mathbf{l} \in \mathbb{H}_{n}^{*}} c_{\mathbf{l}}^{(n)} \phi_{\mathbf{l}}\left(\frac{\mathbf{k}-\mathbf{j}}{4 n}\right) .
$$

Since $\Omega_{H}$ tiles $\mathbb{R}_{H}^{4}$, there exist $\mathbf{m}, \mathbf{l} \in \mathbb{Z}_{H}^{4}$ such that $\frac{\mathbf{m}}{4 n} \in \Omega_{H}$ and $\mathbf{k}-\mathbf{j}=\mathbf{m}+4 n \mathbf{l}$. Thus, by Theorem 3.15 .

$$
\begin{aligned}
\ell_{\mathbf{j}, n}\left(\frac{\mathbf{k}}{4 n}\right) & =\frac{1}{4 n^{3}} \sum_{\mathbf{i} \in \mathbb{H}_{n}^{*}} c_{\mathbf{i}}^{(n)} \phi_{\mathbf{i}}\left(\frac{\mathbf{m}}{4 n}\right)=\frac{1}{4 n^{3}} \sum_{\mathbf{i} \in \mathbb{H}_{n}^{*}} c_{\mathbf{i}}^{(n)} \phi_{\mathbf{m}}\left(\frac{\mathbf{i}}{4 n}\right) \\
& =\left\langle\phi_{\mathbf{m}}, \phi_{0}\right\rangle_{n}^{*}=\left\langle\phi_{\mathbf{m}}, \phi_{0}\right\rangle=\delta_{\mathbf{m}, 0}
\end{aligned}
$$

Equivalently we can write the above equation as

$$
\ell_{\mathbf{j}, n}\left(\frac{\mathbf{k}}{4 n}\right)=\left\langle\phi_{\mathbf{k}}, \phi_{\mathbf{j}}\right\rangle_{n}^{*}= \begin{cases}1, & \mathbf{k}=\mathbf{j}+4 n \mathbf{l}, \mathbf{l} \in \mathbb{Z}_{\mathbb{H}}^{4}, \\ 0, & \text { otherwise }\end{cases}
$$

from which (3.23) follows. 
To derive the compact formula for $\ell_{\mathbf{j}, n}$ we start with the obvious fact that $\partial \mathbb{H}_{n}^{*}=$ $\mathbb{H}_{n}^{*} \backslash \mathbb{H}_{n-1}^{*}$, so that

$$
\begin{aligned}
\sum_{\mathbf{k} \in \partial \mathbb{H}_{n}^{*}} c_{\mathbf{k}}^{(n)} \phi_{\mathbf{k}}(\mathbf{t}) & =\frac{1}{2} \sum_{\mathbf{k} \in \mathbb{H}_{n}^{*} \backslash \mathbb{H}_{n-1}^{*}} \phi_{\mathbf{k}}(\mathbf{t})-\sum_{\mathbf{k} \in \partial \mathbb{H}_{n}^{*}}\left(\frac{1}{2}-c_{\mathbf{k}}^{(n)}\right) \phi_{\mathbf{k}}(\mathbf{t}) \\
& =\frac{1}{2}\left(D_{n}^{H}(\mathbf{t})-D_{n-1}^{H}(\mathbf{t})\right)-\sum_{\mathbf{k} \in \partial \mathbb{H}_{n}^{*}}\left(\frac{1}{2}-c_{\mathbf{k}}^{(n)}\right) \phi_{\mathbf{k}}(\mathbf{t}) .
\end{aligned}
$$

Since $\mathbb{H}_{n}^{*}=\mathbb{H}_{n}^{\circ} \cup \partial \mathbb{H}_{n}^{*}$, we then derive from the decomposition of $\partial \mathbb{H}_{n}^{*}$ into $\mathbb{H}_{n}^{i, j}$ and the values of $c_{\mathbf{k}}^{(n)}$ that

$$
\begin{aligned}
\Phi_{n}^{*}(\mathbf{t})=\frac{1}{4 n^{3}}[ & \frac{1}{2}\left(D_{n}^{H}(\mathbf{t})+D_{n-1}^{H}(\mathbf{t})\right)-\frac{1}{6} \sum_{k \in \mathbb{H}_{n}^{1,2} \cup \mathbb{H}_{n}^{2,1}} \phi_{\mathbf{k}}(\mathbf{t}) \\
& \left.-\frac{1}{4} \sum_{k \in \mathbb{H}_{n}^{1,3} \cup \mathbb{H}_{n}^{3,1}} \phi_{\mathbf{k}}(\mathbf{t})-\frac{1}{3} \sum_{k \in \mathbb{H}_{n}^{2,2}} \phi_{\mathbf{k}}(\mathbf{t})\right] .
\end{aligned}
$$

Let us define $\mathbb{H}_{n}^{I, J}:=\left\{\mathbf{k} \in \mathbb{H}: \frac{\mathbf{k}}{4 n} \in B_{I, J}\right\}$ for $I, J \subset \mathbb{N}_{4}$ and also define $\left[\mathbb{H}_{n}^{I, J}\right]:=$ $\left\{\mathbf{k} \in \mathbb{H}: \frac{\mathbf{k}}{4 n} \in\left[B_{I, J}\right]\right\}$. It follows from (3.12), (3.21) and Lemma 3.8 that

$$
\mathbb{H}_{n}^{i, j}=\bigcup_{I, J \in \mathcal{K}_{0}^{i, j}}\left[\mathbb{H}_{n}^{I, J}\right] \quad \text { and } \quad\left[\mathbb{H}_{n}^{I, J}\right]=\bigcup_{\sigma \in \mathcal{G}_{I \cup J}} \mathbb{H}_{n}^{I, J} \sigma .
$$

In particular, by (3.13), we have

$$
\begin{aligned}
& \mathbb{H}_{n}^{1,2}=\left[\mathbb{H}_{n}^{\{1\},\{2,3\}}\right] \cup\left[\mathbb{H}_{n}^{\{1\},\{2,4\}}\right] \cup\left[\mathbb{H}_{n}^{\{1\},\{3,4\}}\right] \cup\left[\mathbb{H}_{n}^{\{2\},\{3,4\}}\right], \\
& \mathbb{H}_{n}^{2,1}=\left[\mathbb{H}_{n}^{\{1,2\},\{3\}}\right] \cup\left[\mathbb{H}_{n}^{\{1,2\},\{4\}}\right] \cup\left[\mathbb{H}_{n}^{\{1,3\},\{4\}}\right] \cup\left[\mathbb{H}_{n}^{\{2,3\},\{4\}}\right] .
\end{aligned}
$$

By (3.10), $\left[\mathbb{H}_{n}^{\{1\},\{2,3\}}\right]=\mathbb{H}_{n}^{\{1\},\{2,3\}} \cup \mathbb{H}_{n}^{\{2\},\{1,3\}} \cup \mathbb{H}_{n}^{\{3\},\{1,2\}}$. Furthermore, it follows from (3.14) that $\left[\mathbb{H}_{n}^{\{1\},\{2,4\}}\right]=\left[\mathbb{H}_{n}^{\{1\},\{2,3\}}\right] \sigma_{34},\left[\mathbb{H}_{n}^{\{1\},\{3,4\}}\right]=\left[\mathbb{H}_{n}^{\{1\},\{2,3\}}\right] \sigma_{24}$, $\left[\mathbb{H}_{n}^{\{2\},\{3,4\}}\right]=\left[\mathbb{H}_{n}^{\{1\},\{2,3\}}\right] \sigma_{12} \sigma_{24}$. Using the explicit formulas in (3.9), it is easy to see that

$$
\mathbb{H}_{n}^{\{1\},\{2,3\}}=\{(j+2 n, j-2 n, j-2 n, 2 n-3 j): 1 \leq j \leq n-1\} .
$$

Consequently, using $t_{1}+t_{2}+t_{3}+t_{4}=0$, it follows readily that

$$
\begin{aligned}
\sum_{\mathbf{k} \in\left[\mathbb{H}_{n}^{\{1\},\{2,3\}}\right]} \phi_{\mathbf{k}}(\mathbf{t}) & =\sum_{\mathbf{k} \in \mathbb{H}_{n}^{\{1\},\{2,3\}}} \phi_{\mathbf{k}}(\mathbf{t})+\sum_{\mathbf{k} \in \mathbb{H}_{n}^{\{2\},\{1,3\}}} \phi_{\mathbf{k}}(\mathbf{t})+\sum_{\mathbf{k} \in \mathbb{H}_{n}^{\{3\},\{1,2\}}} \phi_{\mathbf{k}}(\mathbf{t}) \\
& =\sum_{j=1}^{n-1} e^{-2 \pi i j t_{4}}\left(e^{2 n \pi i\left(t_{1}+t_{4}\right)}+e^{2 n \pi i\left(t_{2}+t_{4}\right)}+e^{2 n \pi i\left(t_{3}+t_{4}\right)}\right) \\
& =\frac{\sin (n-1) \pi t_{4}}{\sin \pi t_{4}}\left(e^{n \pi i\left(2 t_{1}+t_{4}\right)}+e^{n \pi i\left(2 t_{2}+t_{4}\right)}+e^{n \pi i\left(2 t_{3}+t_{4}\right)}\right),
\end{aligned}
$$

where in the last step the sum is evaluated using (3.20). The explicit formulas for other components of $\sum_{\mathbf{k} \in \mathbb{H}_{n}^{1,2}} \phi_{\mathbf{k}}$ follow from the above expression by permuting the variables. In a similar manner, we have $\left[\mathbb{H}_{n}^{\{1,2\},\{3\}}\right]=\mathbb{H}_{n}^{\{1,2\},\{3\}} \cup \mathbb{H}_{n}^{\{1,3\},\{2\}} \cup$ $\mathbb{H}_{n}^{\{2,3\},\{1\}}$, and, using (3.14) again, $\left[\mathbb{H}_{n}^{\{1,2\},\{4\}}\right]=\left[\mathbb{H}_{n}^{\{1,2\},\{3\}}\right] \sigma_{34},\left[\mathbb{H}_{n}^{\{1,3\},\{4\}}\right]=$ $\left[\mathbb{H}_{n}^{\{1,2\},\{3\}}\right] \sigma_{23} \sigma_{34},\left[\mathbb{H}_{n}^{\{2,3\},\{4\}}\right]=\left[\mathbb{H}_{n}^{\{1,2\},\{3\}}\right] \sigma_{13} \sigma_{34}$. Moreover, we also have

$$
\mathbb{H}_{n}^{\{1,2\},\{3\}}=\{(2 n-j, 2 n-j,-2 n-j,-2 n+3 j): 1 \leq j \leq n-1\} .
$$


Thus, using $t_{1}+t_{2}+t_{3}+t_{4}=0$, we can deduce as before that

$$
\begin{aligned}
\sum_{\mathbf{k} \in\left[\mathbb{H}_{n}^{\{1,2\},\{3\}}\right]} \phi_{\mathbf{k}}(\mathbf{t}) & =\sum_{\mathbf{k} \in \mathbb{H}_{n}^{\{1,2\},\{3\}}} \phi_{\mathbf{k}}(\mathbf{t})+\sum_{\mathbf{k} \in \mathbb{H}_{n}^{\{1,3\},\{2\}}} \phi_{\mathbf{k}}(\mathbf{t})+\sum_{\mathbf{k} \in \mathbb{H}_{n}^{\{2,3\},\{1\}}} \phi_{\mathbf{k}}(\mathbf{t}) \\
& =\frac{\sin (n-1) \pi t_{4}}{\sin \pi t_{4}}\left(e^{-n \pi i\left(2 t_{1}+t_{4}\right)}+e^{-n \pi i\left(2 t_{2}+t_{4}\right)}+e^{-n \pi i\left(2 t_{3}+t_{4}\right)}\right),
\end{aligned}
$$

from which the explicit formulas of other components of $\sum_{\mathbf{k} \in \mathbb{H}_{n}^{2,1}} \phi_{\mathbf{k}}$ follow from permuting the variables.

Putting the sums over $\mathbb{H}_{n}^{1,2}$ and $\mathbb{H}_{n}^{2,1}$ together, we obtain

$$
\sum_{\mathbf{k} \in \mathbb{H}_{n}^{1,2} \cup \mathbb{H}_{n}^{2,1}} \phi_{k}(\mathbf{t})=2 \sum_{\nu=1}^{4} \frac{\sin (n-1) \pi t_{\nu}}{\sin \pi t_{\nu}} \sum_{\substack{j=1 \\ j \neq \nu}}^{4} \cos n \pi\left(2 t_{j}+t_{\nu}\right) .
$$

Using (3.15), it is easy to see that $\mathbb{H}_{n}^{1,3}=\{(n, n, n,-3 n) \sigma: \sigma \in \mathcal{G}\}, \mathbb{H}_{n}^{2,2}=$ $\{(2 n, 2 n,-2 n,-2 n) \sigma: \sigma \in \mathcal{G}\}$ and $\mathbb{H}_{n}^{3,1}=\{(3 n,-n,-n,-n) \sigma: \sigma \in \mathcal{G}\}$, from which it follows that

$$
\sum_{\mathbf{k} \in \mathbb{H}_{n}^{1,3} \cup \mathbb{H}_{n}^{3,1}} \phi_{k}(\mathbf{t})=\sum_{j=1}^{4}\left(e^{2 \pi i n t_{j}}+e^{-2 \pi i n t_{j}}\right)=2 \sum_{j=1}^{4} \cos 2 \pi n t_{j} .
$$

Furthermore, using $t_{1}+t_{2}+t_{3}+t_{4}=0$, it is easy to see that

$$
\sum_{\mathbf{k} \in \mathbb{H}_{n}^{2,2}} \phi_{\mathbf{k}}(\mathbf{t})=\sum_{1 \leq \mu<\nu \leq 4} e^{2 \pi i n\left(t_{\mu}+t_{\nu}\right)}=\sum_{1 \leq \mu<\nu \leq 4} \cos 2 \pi n\left(t_{\mu}+t_{\nu}\right) .
$$

Putting these terms into (3.26) completes the proof.

The compact formula of the interpolation function allows us to estimate the operator norm of $I_{n}^{*}$, which is usually referred to as the Lebesgue constant.

Theorem 3.19. Let $\left\|I_{n}^{*}\right\|_{\infty}$ denote the operator norm of $I_{n}^{*}: C\left(\bar{\Omega}_{H}\right) \mapsto C\left(\bar{\Omega}_{H}\right)$. Then there is a constant $c$, independent of $n$, such that

$$
\left\|I_{n}^{*}\right\|_{\infty} \leq c(\log n)^{3} .
$$

Proof. A standard procedure shows that

$$
\left\|I_{n}^{*}\right\|_{\infty}=\max _{\mathbf{t} \in \bar{\Omega}_{H}} \sum_{\mathbf{k} \in \mathbb{H}_{n}^{*}}\left|\Phi_{n}^{*}\left(\mathbf{t}-\frac{\mathbf{k}}{4 n}\right)\right| .
$$

Using the compact formula of $\Phi_{n}^{*}$ in Theorem 3.18, it is easy to see that it suffices to prove that

$$
\frac{1}{4 n^{3}} \max _{\mathbf{t} \in \bar{\Omega}_{H}} \sum_{\mathbf{k} \in \mathbb{H}_{n}^{*}}\left|D_{n}^{H}\left(\mathbf{t}-\frac{\mathbf{k}}{4 n}\right)\right| \leq c(\log n)^{3}, \quad n \geq 0 .
$$

Furthermore, as in the proof of Theorem 3.11, the formula of $D_{n}^{H}$ in (3.19) shows that our main task is to establish the estimate

$$
I_{\{1,2,3\}}:=\frac{1}{4 n^{3}} \max _{\mathbf{t} \in \bar{\Omega}_{H}} \sum_{\mathbf{k} \in \mathbb{H}_{n}^{*}}\left|K_{n}\left(t_{1}-\frac{k_{1}}{4 n}\right) K_{n}\left(t_{2}-\frac{k_{2}}{4 n}\right) K_{n}\left(t_{3}-\frac{k_{3}}{4 n}\right)\right| \leq c(\log n)^{3},
$$


and three other similar estimates $I_{\{1,2,4\}}, I_{\{1,3,4\}}$ and $I_{\{2,3,4\}}$, respectively. Enlarging the domain $\mathbb{H}_{n}^{*}$ to $\left\{\mathbf{k} \in \mathbb{Z}_{H}^{4}:-4 n \leq k_{i} \leq 4 n, k_{i}=0(\bmod 4), 1 \leq i \leq 3\right\}$, we see that

$$
\begin{aligned}
I_{\{1,2,3\}} & \leq \frac{1}{4 n^{3}} \max _{t \in[-1,1]^{3}} \sum_{k_{1}=-n}^{n} \sum_{k_{2}=-n}^{n} \sum_{k_{3}=-n}^{n}\left|K_{n}\left(t_{1}-\frac{k_{1}}{n}\right) K_{n}\left(t_{2}-\frac{k_{2}}{n}\right) K_{n}\left(t_{3}-\frac{k_{3}}{n}\right)\right| \\
& \leq \frac{1}{4} \max _{t \in[-1,1]}\left(\frac{1}{n} \sum_{k=-n}^{n}\left|\frac{\sin (n+1) \pi\left(t-\frac{k}{n}\right)}{\sin \pi\left(t-\frac{k}{n}\right)}\right|\right)^{3} \leq c(\log n)^{3},
\end{aligned}
$$

where the last step follows from the standard estimate of one variable (cf. [13, Vol. II, p. 19]).

Again we expect that the estimate is sharp, that is, $\left\|I_{n}^{*}\right\| \geq c(\log n)^{3}$; and the problem is again that there is a sum of four terms of the same type.

\section{Discrete Fourier Analysis on the tetrahedron}

Considering functions invariant under the isometrics of the fcc lattice, the discrete Fourier analysis on the dodecahedron in the previous section can be carried over to the analysis on the tetrahedron.

4.1. Generalized sine and cosine functions. The fcc lattice is the root lattice of the reflection group $\mathcal{A}_{3}$ 2, Chap. 4]. Under the homogeneous coordinates, the group $\mathcal{A}_{3}$ is generated by the reflections $\left\{\sigma_{i j}: 1 \leq i<j \leq 4\right\}$, where $\sigma_{i j}$ is the reflection defined by $\mathbf{t} \sigma_{i j}=\mathbf{t}-2 \frac{\left\langle\mathbf{t}, \mathbf{e}_{i, j}\right\rangle}{\left\langle\mathbf{e}_{i, j}, \mathbf{e}_{i, j}\right\rangle} \mathbf{e}_{i, j}$ with $\mathbf{e}_{i, j}=e_{i}-e_{j}$ as before. Thus $\mathcal{A}_{3}$ is the permutation group in the previous section. Denote the identity element in $\mathcal{A}_{3}$ by 1 . It is easy to see that we have

$$
\sigma_{i j}^{2}=1, \quad \sigma_{i j} \sigma_{j k} \sigma_{i j}=\sigma_{i k}, \quad i, j, k \in \mathbb{N}_{4} .
$$

For $\sigma \in \mathcal{G}=\mathcal{A}_{3}$, let $|\sigma|$ denote the number of inversions in $\sigma$. The group $\mathcal{G}$ is naturally divided into two parts, $\mathcal{G}^{+}:=\{\sigma \in \mathcal{G}:|\sigma| \equiv 0(\bmod 2)\}$ of elements with even inversions, and $\mathcal{G}^{-}:=\{\sigma \in \mathcal{G}:|\sigma| \equiv 1(\bmod 2)\}$ of elements with odd inversions. Writing it out explicitly, we have

$$
\begin{aligned}
& \mathcal{G}^{+}=\left\{1, \sigma_{12} \sigma_{13}, \sigma_{13} \sigma_{12}, \sigma_{12} \sigma_{14}, \sigma_{14} \sigma_{12}, \sigma_{13} \sigma_{14},\right.\left.\sigma_{14} \sigma_{13}, \sigma_{23} \sigma_{24}, \sigma_{24} \sigma_{23}, \sigma_{12} \sigma_{34}, \sigma_{13} \sigma_{24}, \sigma_{14} \sigma_{23}\right\} \\
& \mathcal{G}^{-}=\left\{\sigma_{12}, \sigma_{13}, \sigma_{14}, \sigma_{23}, \sigma_{24}, \sigma_{34}, \sigma_{12} \sigma_{13} \sigma_{14}, \sigma_{12} \sigma_{14} \sigma_{13},\right. \\
&\left.\sigma_{13} \sigma_{12} \sigma_{14}, \sigma_{13} \sigma_{14} \sigma_{12}, \sigma_{14} \sigma_{12} \sigma_{13}, \sigma_{14} \sigma_{13} \sigma_{12}\right\} .
\end{aligned}
$$

The action of $\sigma \in \mathcal{G}$ on the function $f: \mathbb{R}_{H}^{4} \mapsto \mathbb{R}$ is defined by $\sigma f(\mathbf{t}):=f(\mathbf{t} \sigma)$. A function $f$ in homogeneous coordinates is called invariant under $\mathcal{G}$ if $\sigma f=f$ for all $\sigma \in \mathcal{G}$, and it is called anti-invariant under $\mathcal{G}$ if $\sigma f=\rho(\sigma) f$ with $\rho(\sigma)=1$ if $\sigma \in \mathcal{G}^{+}$and $\rho(\sigma)=-1$ if $\sigma \in \mathcal{G}^{-}$.

The following proposition follows immediately from the definition.

Proposition 4.1. Define two operator $\mathcal{P}^{+}$and $\mathcal{P}^{-}$acting on $f(\mathbf{t})$ by

$$
\mathcal{P}^{ \pm} f(\mathbf{t}):=\frac{1}{24}\left[\sum_{\sigma \in \mathcal{G}^{+}} f(\mathbf{t} \sigma) \pm \sum_{\sigma \in \mathcal{G}^{-}} f(\mathbf{t} \sigma)\right]
$$


Then the operators $\mathcal{P}^{+}$and $\mathcal{P}^{-}$are projections from the class of $H$-periodic functions onto the class of invariant, and respectively anti-invariant functions.

Applying the operators $\mathcal{P}^{ \pm}$to $\phi_{\mathbf{k}}(\mathbf{t})=e^{\frac{\pi i}{2} \mathbf{k} \cdot \mathbf{t}}$ gives basic invariant and antiinvariant functions, which we denote by $\mathrm{TC}_{\mathbf{k}}$ and $\mathrm{TS}_{\mathbf{k}}$, respectively, as they are analogues of cosine and sine functions. We formerly define them as follows.

Definition 4.2. For $\mathrm{k} \in \mathbb{H}$ define

$$
\begin{aligned}
& \mathrm{TC}_{\mathbf{k}}(\mathbf{t}):=\mathcal{P}^{+} \phi_{\mathbf{k}}(\mathbf{t})=\frac{1}{24}\left[\sum_{\sigma \in \mathcal{G}^{+}} \phi_{\mathbf{k}}(\mathbf{t} \sigma)+\sum_{\sigma \in \mathcal{G}^{-}} \phi_{\mathbf{k}}(\mathbf{t} \sigma)\right], \\
& \mathrm{TS}_{\mathbf{k}}(\mathbf{t}):=-\mathcal{P}^{-} \phi_{\mathbf{k}}(\mathbf{t})=-\frac{1}{24}\left[\sum_{\sigma \in \mathcal{G}^{+}} \phi_{\mathbf{k}}(\mathbf{t} \sigma)-\sum_{\sigma \in \mathcal{G}^{-}} \phi_{\mathbf{k}}(\mathbf{t} \sigma)\right],
\end{aligned}
$$

and call them generalized cosine and generalized sine, respectively.

Evidently $\mathrm{TC}_{\mathbf{k}}$ is invariant and $\mathrm{TS}_{\mathbf{k}}$ is anti-invariant. The rhombic dodecahedron is invariant under our group $\mathcal{G}$ of order 24 ; its fundamental domain is a tetrahedron. For invariant functions, we can make use of symmetry to translate results on the rhombic dodecahedron to one of its 24 tetrahedrons. We shall choose our reference tetrahedron as

$$
\triangle:=\left\{x \in \mathbb{R}^{3}: 0 \leq x_{3} \pm x_{2}, x_{2} \pm x_{1} \leq 1\right\} .
$$

In the homogeneous coordinates, by (3.2), this tetrahedron becomes

$$
\triangle_{H}:=\left\{\mathbf{t} \in \mathbb{R}_{H}^{4}: 0 \leq t_{1}-t_{2}, t_{2}-t_{3}, t_{3}-t_{4}, t_{1}-t_{4} \leq 1\right\} .
$$

See Figure 4.1 below, in which coordinates of the corners are given in both $\mathbb{R}^{3}$ coordinates and homogeneous coordinates in $\mathbb{R}^{4}$.

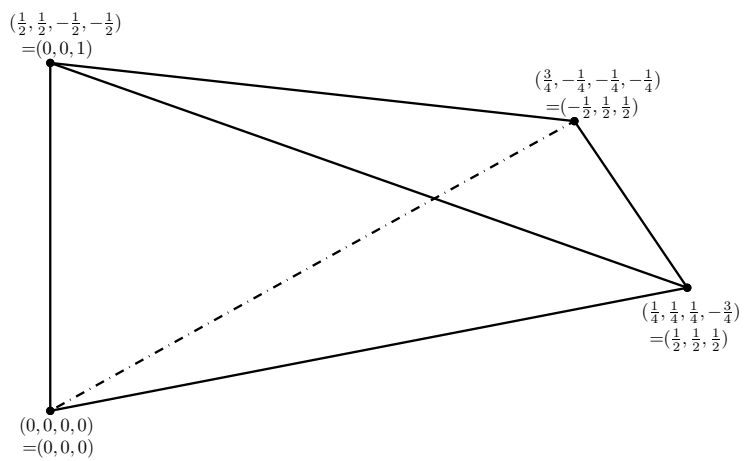

FiguRE 4.1. Reference tetrahedron.

When $\mathrm{TC}_{\mathbf{k}}$ are restricted to the tetrahedron $\triangle_{H}$, we only need to consider a subset of $\mathbf{k} \in \mathbb{H}$. In fact, it is easy to see that

$$
\mathrm{TC}_{\mathbf{k} \sigma}(\mathbf{t})=\mathrm{TC}_{\mathbf{k}}(\mathbf{t} \sigma)=\mathrm{TC}_{\mathbf{k}}(\mathbf{t})
$$

for $\mathbf{t} \in \triangle_{H}$ and $\sigma \in \mathcal{G}$. Thus, we can restrict $\mathbf{k}$ to the index set

$$
\Lambda:=\left\{\mathbf{k} \in \mathbb{H}: k_{1} \geq k_{2} \geq k_{3} \geq k_{4}\right\},
$$

when we consider $\mathrm{TC}_{\mathbf{k}}$. As for $\mathrm{TS}_{\mathbf{k}}$, it is easy to see that $\mathrm{TS}_{\mathbf{k} \sigma}(\mathbf{t})=\mathrm{TS}_{\mathbf{k}}(\mathbf{t} \sigma)=$ $\mathrm{TS}_{\mathbf{k}}(\mathbf{t})$ for $\sigma \in \mathcal{G}^{+}$and $\mathrm{TS}_{\mathbf{k} \sigma}(\mathbf{t})=\mathrm{TS}_{\mathbf{k}}(\mathbf{t} \sigma)=-\mathrm{TS}_{\mathbf{k}}(\mathbf{t})$ for $\sigma \in \mathcal{G}^{-}$. In particular, 
$\mathrm{TS}_{\mathbf{k}}(\mathbf{t})=0$ whenever two or more components of $\mathbf{k}$ are equal. Thus $\mathrm{TS}_{\mathbf{k}}$ are defined only for $\mathbf{k} \in \Lambda^{\circ}$, where

$$
\Lambda^{\circ}:=\left\{\mathbf{k} \in \mathbb{H}: k_{1}>k_{2}>k_{3}>k_{4}\right\},
$$

which is the set of the interior points of $\Lambda$. Since $\mathbf{k} \in \mathbb{H}$ implies that $k_{i} \in \mathbb{Z}$ and $k_{1}+k_{2}+k_{3}+k_{4}=0$, the points in $\Lambda$ lie in a three dimensional wedge. To describe the points on the boundary of $\Lambda$, we further define

$$
\begin{aligned}
\Lambda^{f} & :=\left\{\left(\mathbf{k} \in \mathbb{H}: k_{1}=k_{2}>k_{3}>k_{4} \text { or } k_{1}>k_{2}=k_{3}>k_{4} \text { or } k_{1}>k_{2}>k_{3}=k_{4}\right\},\right. \\
\Lambda^{e, 2} & :=\{(k, k, k,-3 k),(3 k,-k,-k,-k): k>0\}, \\
\Lambda^{e, 1} & :=\{(2 k, 2 k,-2 k,-2 k): k>0\}, \quad \Lambda^{v}:=\{(0,0,0,0)\} .
\end{aligned}
$$

Then evidently $\Lambda \backslash \Lambda^{\circ}=\Lambda^{f} \cup \Lambda^{e, 1} \cup \Lambda^{e, 2} \cup \Lambda^{v}$.

Let $\mathbf{k} \mathcal{G}$ denote the orbit of $\mathbf{k}$ under $\mathcal{G}$, that is, $\mathbf{k} \mathcal{G}:=\{\mathbf{k} \sigma: \sigma \in \mathcal{G}\}$. Then, for $\mathbf{k}, \mathbf{j} \in \Lambda, \mathbf{k} \mathcal{G} \cap \mathbf{j} \mathcal{G}=\emptyset$ whenever $\mathbf{k} \neq \mathbf{j}$. Furthermore, it is easy to see that

$$
\mathrm{TC}_{\mathbf{k}}(\mathbf{t})=\frac{1}{|\mathbf{k} \mathcal{G}|} \sum_{\mathbf{j} \in \mathbf{k} \mathcal{G}} \phi_{\mathbf{j}}, \quad|\mathbf{k} \mathcal{G}|= \begin{cases}24, & \mathbf{k} \in \Lambda^{\circ}, \\ 12, & \mathbf{k} \in \Lambda^{f}, \\ 6, & \mathbf{k} \in \Lambda^{e, 1}, \\ 4, & \mathbf{k} \in \Lambda^{e, 2}, \\ 1, & \mathbf{k}=0 \in \Lambda^{v}\end{cases}
$$

We define an inner product on $\triangle_{H}$ by

$$
\langle f, g\rangle_{\triangle_{H}}:=\frac{1}{\left|\triangle_{H}\right|} \int_{\triangle_{H}} f(\mathbf{t}) \overline{g(\mathbf{t})} d \mathbf{t}=12 \int_{\triangle_{H}} f(\mathbf{t}) \overline{g(\mathbf{t})} d t_{1} d t_{2} d t_{3} .
$$

If $f \bar{g}$ is invariant under $\mathcal{G}$, then it follows immediately that $\langle f, g\rangle=\langle f, g\rangle_{\triangle_{H}}$. Furthermore, the generalized cosine and sine functions are orthogonal with respect to this inner product.

Proposition 4.3. For $\mathbf{k}, \mathbf{j} \in \Lambda$,

$$
\left\langle\mathrm{TC}_{\mathbf{k}}, \mathrm{TC}_{\mathbf{j}}\right\rangle_{\triangle_{H}}=\frac{\delta_{\mathbf{k}, \mathbf{l}}}{|\mathbf{k} \mathcal{G}|}=\delta_{\mathbf{k}, 1} \begin{cases}1, & \mathbf{k}=0 \\ \frac{1}{4}, & \mathbf{k} \in \Lambda^{e, 2} \\ \frac{1}{6}, & \mathbf{k} \in \Lambda^{e, 1} \\ \frac{1}{12}, & \mathbf{k} \in \Lambda^{f} \\ \frac{1}{24}, & \mathbf{k} \in \Lambda^{\circ}\end{cases}
$$

for $\mathbf{k}, \mathbf{j} \in \Lambda^{\circ}$,

$$
\left\langle\mathrm{TS}_{\mathbf{k}}, \mathrm{TS}_{\mathbf{j}}\right\rangle_{\triangle_{H}}=\frac{1}{24} \delta_{\mathbf{k}, \mathbf{j}}
$$

Proof. Both of these relations follow from the identity $\langle f, g\rangle=\langle f, g\rangle_{\triangle_{H}}$ for invariant functions. For (4.5), the invariance is evident and we only have to use the orthogonality of $\phi_{\mathbf{k}}$ in Proposition 3.1 and (4.4). For (4.6), we use the fact that $\mathrm{TS}_{\mathbf{k}}(\mathbf{t}) \overline{\mathrm{TS}_{\mathbf{j}}(\mathbf{t})}$ is invariant under $\mathcal{G}$ and the orthogonality of $\phi_{\mathbf{k}}$ on $\Omega_{H}$. 
We can derive more explicit formulas for the generalized cosine and sine functions by making use of the homogeneous coordinates. For example, we have

$$
\begin{aligned}
& \phi_{\mathbf{k}}\left(t_{1}, t_{2}, t_{3}, t_{4}\right)-\phi_{\mathbf{k}}\left(t_{2}, t_{1}, t_{3}, t_{4}\right)-\phi_{\mathbf{k}}\left(t_{1}, t_{2}, t_{4}, t_{3}\right)+\phi_{\mathbf{k}}\left(t_{2}, t_{1}, t_{4}, t_{3}\right) \\
& =\left[e^{\frac{\pi i}{2}\left(k_{1} t_{1}+k_{2} t_{2}\right)}-e^{\frac{\pi i}{2}\left(k_{1} t_{2}+k_{2} t_{1}\right)}\right]\left[e^{\frac{\pi i}{2}\left(k_{3} t_{3}+k_{4} t_{4}\right)}-e^{\left.\frac{\pi i}{2}\left(k_{3} t_{4}+k_{4} t_{3}\right)\right]}\right. \\
& =2 i e^{\frac{\pi i}{4}\left(k_{1}+k_{2}\right)\left(t_{1}+t_{2}\right)} \sin \frac{\pi\left(k_{1}-k_{2}\right)\left(t_{1}-t_{2}\right)}{4} \cdot 2 i e^{\frac{\pi i}{4}\left(k_{3}+k_{4}\right)\left(t_{3}+t_{4}\right)} \sin \frac{\pi\left(k_{3}-k_{4}\right)\left(t_{3}-t_{4}\right)}{4} \\
& =-4 e^{\frac{\pi i}{4}\left[\left(k_{1}+k_{2}\right)\left(t_{1}+t_{2}\right)+\left(k_{3}+k_{4}\right)\left(t_{3}+t_{4}\right)\right]} \sin \frac{\pi\left(k_{1}-k_{2}\right)\left(t_{1}-t_{2}\right)}{4} \sin \frac{\pi\left(k_{3}-k_{4}\right)\left(t_{3}-t_{4}\right)}{4} \\
& =\phi_{\mathbf{k}}\left(t_{3}, t_{4}, t_{1}, t_{2}\right)-\phi_{\mathbf{k}}\left(t_{4}, t_{3}, t_{1}, t_{2}\right)-\phi_{\mathbf{k}}\left(t_{3}, t_{4}, t_{2}, t_{1}\right)+\phi_{\mathbf{k}}\left(t_{4}, t_{3}, t_{2}, t_{1}\right) .
\end{aligned}
$$

Similarly or by permuting the variables, we also have,

$$
\begin{aligned}
& \phi_{\mathbf{k}}\left(t_{1}, t_{3}, t_{4}, t_{2}\right)-\phi_{\mathbf{k}}\left(t_{3}, t_{1}, t_{4}, t_{2}\right)-\phi_{\mathbf{k}}\left(t_{1}, t_{3}, t_{2}, t_{4}\right)+\phi_{\mathbf{k}}\left(t_{3}, t_{1}, t_{2}, t_{4}\right) \\
& =\phi_{\mathbf{k}}\left(t_{4}, t_{2}, t_{1}, t_{3}\right)-\phi_{\mathbf{k}}\left(t_{4}, t_{2}, t_{3}, t_{1}\right)-\phi_{\mathbf{k}}\left(t_{2}, t_{4}, t_{1}, t_{3}\right)+\phi_{\mathbf{k}}\left(t_{2}, t_{4}, t_{3}, t_{1}\right) \\
& =-4 e^{\frac{\pi i}{4}\left[\left(k_{1}+k_{2}\right)\left(t_{1}+t_{3}\right)+\left(k_{3}+k_{4}\right)\left(t_{2}+t_{4}\right)\right]} \sin \frac{\pi\left(k_{1}-k_{2}\right)\left(t_{1}-t_{3}\right)}{4} \sin \frac{\pi\left(k_{3}-k_{4}\right)\left(t_{4}-t_{2}\right)}{4},
\end{aligned}
$$

and

$$
\begin{aligned}
& \phi_{\mathbf{k}}\left(t_{1}, t_{4}, t_{2}, t_{3}\right)-\phi_{\mathbf{k}}\left(t_{4}, t_{1}, t_{2}, t_{3}\right)-\phi_{\mathbf{k}}\left(t_{1}, t_{4}, t_{3}, t_{2}\right)+\phi_{\mathbf{k}}\left(t_{4}, t_{1}, t_{3}, t_{2}\right) \\
& =\phi_{\mathbf{k}}\left(t_{2}, t_{3}, t_{1}, t_{4}\right)-\phi_{\mathbf{k}}\left(t_{2}, t_{3}, t_{4}, t_{1}\right)-\phi_{\mathbf{k}}\left(t_{3}, t_{2}, t_{1}, t_{4}\right)+\phi_{\mathbf{k}}\left(t_{3}, t_{2}, t_{4}, t_{1}\right) \\
& =-4 e^{\frac{\pi i}{4}\left[\left(k_{1}+k_{2}\right)\left(t_{1}+t_{4}\right)+\left(k_{3}+k_{4}\right)\left(t_{2}+t_{3}\right)\right]} \sin \frac{\pi\left(k_{1}-k_{2}\right)\left(t_{1}-t_{4}\right)}{4} \sin \frac{\pi\left(k_{3}-k_{4}\right)\left(t_{2}-t_{3}\right)}{4} .
\end{aligned}
$$

Consequently, using the homogeneous relations $t_{1}+t_{2}+t_{3}+t_{4}=0$ and $k_{1}+k_{2}+$ $k_{3}+k_{4}=0$, we can then deduce that

$$
\begin{aligned}
\mathrm{TS}_{\mathbf{k}}(\mathbf{t}) & =\frac{1}{3} e^{\frac{\pi i}{2}\left(k_{1}+k_{2}\right)\left(t_{1}+t_{2}\right)} \sin \frac{\pi}{4}\left(k_{1}-k_{2}\right)\left(t_{1}-t_{2}\right) \sin \frac{\pi}{4}\left(k_{3}-k_{4}\right)\left(t_{3}-t_{4}\right) \\
& +\frac{1}{3} e^{\frac{\pi i}{2}\left(k_{1}+k_{2}\right)\left(t_{1}+t_{3}\right)} \sin \frac{\pi}{4}\left(k_{1}-k_{2}\right)\left(t_{1}-t_{3}\right) \sin \frac{\pi}{4}\left(k_{3}-k_{4}\right)\left(t_{4}-t_{2}\right) \\
& +\frac{1}{3} e^{\frac{\pi i}{2}\left(k_{1}+k_{2}\right)\left(t_{1}+t_{4}\right)} \sin \frac{\pi}{4}\left(k_{1}-k_{2}\right)\left(t_{1}-t_{4}\right) \sin \frac{\pi}{4}\left(k_{3}-k_{4}\right)\left(t_{2}-t_{3}\right) .
\end{aligned}
$$

In a similar way, we obtain

$$
\begin{aligned}
\mathrm{TC}_{\mathbf{k}}(\mathbf{t}) & =\frac{1}{3} e^{\frac{\pi i}{2}\left(k_{1}+k_{2}\right)\left(t_{1}+t_{2}\right)} \cos \frac{\pi}{4}\left(k_{1}-k_{2}\right)\left(t_{1}-t_{2}\right) \cos \frac{\pi}{4}\left(k_{3}-k_{4}\right)\left(t_{3}-t_{4}\right) \\
& +\frac{1}{3} e^{\frac{\pi i}{2}\left(k_{1}+k_{2}\right)\left(t_{1}+t_{3}\right)} \cos \frac{\pi}{4}\left(k_{1}-k_{2}\right)\left(t_{1}-t_{3}\right) \cos \frac{\pi}{4}\left(k_{3}-k_{4}\right)\left(t_{4}-t_{2}\right) \\
& +\frac{1}{3} e^{\frac{\pi i}{2}\left(k_{1}+k_{2}\right)\left(t_{1}+t_{4}\right)} \cos \frac{\pi}{4}\left(k_{1}-k_{2}\right)\left(t_{1}-t_{4}\right) \cos \frac{\pi}{4}\left(k_{3}-k_{4}\right)\left(t_{2}-t_{3}\right) .
\end{aligned}
$$

Permuting variables $t_{1}, t_{2}, t_{3}, t_{4}$ leads to other representations of $\mathrm{TS}_{\mathbf{k}}$ and $\mathrm{TC}_{\mathbf{k}}$.

4.2. Discrete inner product on the tetrahedron. Using the fact that $\mathrm{TC}_{k}$ and $\mathrm{TS}_{k}$ are invariant and anti-invariant under $\mathcal{G}$ and the orthogonality of $\phi_{\mathbf{k}}$ with respect to the symmetric inner product $\langle\cdot, \cdot\rangle_{n}^{*}$, we can deduce a discrete orthogonality for the generalized cosine and sine functions. For this purpose, we define

$$
\Lambda_{n}:=\mathbb{H}_{n}^{*} \cap \Lambda=\left\{\mathbf{k} \in \mathbb{H}: k_{4} \leq k_{3} \leq k_{2} \leq k_{1} \leq k_{4}+4 n\right\}
$$

The point set with $n=4$ and the region is depicted in Figure 4.1. 


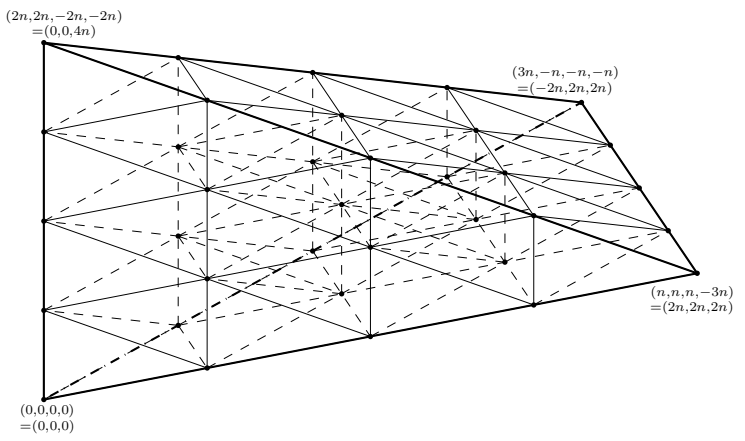

FiguRE $4.2 . \Lambda_{n}$ with $n=4$

By the definition of $\mathbb{H}_{n}^{*}$, the set $\left\{\frac{\mathbf{k}}{4 n}: \mathbf{k} \in \Lambda_{n}\right\}$ contains points inside $\triangle_{H}$. We will also need notation for points on the boundary of $\triangle_{H}$, which are defined as

$$
\begin{aligned}
& \Lambda_{n}^{\circ}:=\mathbb{H}_{n}^{\circ} \cap \Lambda^{\circ}, \quad \Lambda_{n}^{f}:=\left(\Lambda^{f} \cap \mathbb{H}_{n}^{\circ}\right) \cup\left(\Lambda^{\circ} \cap \mathbb{H}_{n}^{1,1}\right), \\
& \Lambda_{n}^{e, 1}:=\left(\Lambda^{e, 1} \cap \mathbb{H}_{n}^{\circ}\right) \cup\left(\Lambda^{f} \cap \mathbb{H}_{n}^{1,1}\right), \\
& \Lambda_{n}^{e, 2}:=\left(\Lambda^{e, 2} \cap \mathbb{H}_{n}^{\circ}\right) \cup\left(\Lambda^{f} \cap \mathbb{H}_{n}^{1,2}\right) \cup\left(\Lambda^{f} \cap \mathbb{H}_{n}^{2,1}\right), \\
& \Lambda_{n}^{v}:=\{0\} \cup\left(\Lambda^{e, 1} \cap \mathbb{H}_{n}^{2,2}\right) \cup\left(\Lambda^{e, 2} \cap \mathbb{H}_{n}^{1,3}\right) \cup\left(\Lambda^{e, 2} \cap \mathbb{H}_{n}^{3,1}\right),
\end{aligned}
$$

corresponding to the set of the interior points, the set of the points on faces, the set of points on two type of edges, and the set of vertices, respectively. More precisely, these sets are given explicitly by

$$
\begin{aligned}
\Lambda_{n}^{\circ}= & \left\{\mathbf{k} \in \mathbb{H}: k_{4}<k_{3}<k_{2}<k_{1}<k_{4}+4 n\right\}, \\
\Lambda_{n}^{f}= & \left\{\mathbf{k} \in \mathbb{H}: k_{4}<k_{3}<k_{2}<k_{1}=k_{4}+4 n \text { or } k_{4}<k_{3}<k_{2}=k_{1}<k_{4}+4 n\right. \\
\left.\quad \text { or } k_{4}<k_{3}=k_{2}<k_{1}<k_{4}+4 n \text { or } k_{4}=k_{3}<k_{2}<k_{1}<k_{4}+4 n\right\}, & \\
\Lambda_{n}^{e, 1}= & \{(2 k, 2 k,-2 k,-2 k),(2 k+n, n-2 k, n-2 k, 2 k-3 n): 0<k<n\}, \\
\Lambda_{n}^{e, 2}= & \{(k, k, k,-3 k),(3 k,-k,-k,-k),(n+k, n+k, n-3 k, k-3 n), \\
(3 n-k, 3 k-n,-n-k,-n-k): 0<k<n\}, & (3 n,-n,-n,-n),(n, n, n,-3 n)\} .
\end{aligned}
$$

We denote by $\mathcal{T} C_{n}$ and $\mathcal{T} S_{n}$ the spaces of the trigonometric polynomials

$$
\mathcal{T} C_{n}:=\operatorname{span}\left\{\mathrm{TC}_{\mathbf{k}}: \mathbf{k} \in \Lambda_{n}\right\}, \quad \mathcal{T} S_{n}:=\operatorname{span}\left\{\mathrm{TS}_{\mathbf{k}}: \mathbf{k} \in \Lambda_{n}^{\circ}\right\}
$$

respectively. We define a discrete inner product $\langle\cdot, \cdot\rangle_{\triangle, n}$ by

$$
\langle f, g\rangle_{\triangle, n}=\frac{1}{4 n^{3}} \sum_{\mathbf{j} \in \Lambda_{n}} \lambda_{\mathbf{j}}^{(n)} f\left(\frac{\mathbf{j}}{4 n} \overline{g\left(\frac{\mathbf{j}}{4 n}\right)},\right.
$$


where

$$
\lambda_{\mathbf{j}}^{(n)}:= \begin{cases}24, & \mathbf{j} \in \Lambda_{n}^{\circ}, \\ 12, & \mathbf{j} \in \Lambda_{n}^{f}, \\ 6, & \mathbf{j} \in \Lambda_{n}^{e, 1}, \\ 4, & \mathbf{j} \in \Lambda_{n}^{e, 2}, \\ 1, & \mathbf{j} \in \Lambda_{n}^{v}\end{cases}
$$

Theorem 4.4. For $f \bar{g} \in \mathcal{T} C_{2 n-1}$,

$$
\langle f, g\rangle_{\triangle_{H}}=\langle f, g\rangle_{\triangle, n}
$$

Moreover, the following cubature formula is exact for all $f \in \mathcal{T C}_{2 n-1}$,

$$
\frac{1}{\left|\triangle_{H}\right|} \int_{\triangle_{H}} f(\mathbf{t}) d \mathbf{t}=\frac{1}{4 n^{3}} \sum_{\mathbf{j} \in \Lambda_{n}} \lambda_{\mathbf{j}}^{(n)} f\left(\frac{\mathbf{j}}{4 n}\right) .
$$

In particular,

$$
\left\langle\mathrm{TC}_{\mathbf{k}}, \mathrm{TC}_{\mathbf{j}}\right\rangle_{\triangle, n}=\frac{\delta_{\mathbf{k}, \mathbf{j}}}{\lambda_{\mathbf{k}}^{(n)}}, \quad \mathbf{k}, \mathbf{j} \in \Lambda_{n}
$$

Proof. We shall deduce the result from Theorem 3.16. Let $f$ be a function invariant under $\mathcal{G}$. Recall the coefficients $c_{\mathbf{j}}^{(n)}$ in the symmetric inner product defined in Definition 3.14. Taking into consideration of the orbits of the points in various regions, we obtain

$$
\begin{aligned}
\sum_{\mathbf{j} \in \mathbb{H}_{n}^{\circ}} c_{\mathbf{j}}^{(n)} f\left(\frac{\mathbf{j}}{4 n}\right)=\sum_{\mathbf{j} \in \mathbb{H}_{n}^{\circ}} f\left(\frac{\mathbf{j}}{4 n}\right) & =24 \sum_{\mathbf{j} \in \Lambda^{\circ} \cap \mathbb{H}_{n}^{\circ}} f\left(\frac{\mathbf{j}}{4 n}\right)+12 \sum_{\mathbf{j} \in \Lambda^{f} \cap \mathbb{H}_{n}^{\circ}} f\left(\frac{\mathbf{j}}{4 n}\right) \\
& +6 \sum_{\mathbf{j} \in \Lambda^{e, 1} \cap \mathbb{H}_{n}^{\circ}} f\left(\frac{\mathbf{j}}{4 n}\right)+4 \sum_{\mathbf{j} \in \Lambda^{e, 2} \cap \mathbb{H}_{n}^{\circ}} f\left(\frac{\mathbf{j}}{4 n}\right)+f(0),
\end{aligned}
$$

and, using the values of $c_{\mathbf{j}}^{(n)}$,

$$
\begin{aligned}
& \sum_{\mathbf{j} \in \mathbb{H}_{n}^{*} \backslash \mathbb{H}_{n}^{\circ}} c_{\mathbf{j}}^{(n)} f\left(\frac{\mathbf{j}}{4 n}\right)=24 \sum_{\mathbf{j} \in \Lambda^{\circ} \cap \mathbb{H}_{n}^{1,1}} c_{\mathbf{j}}^{(n)} f\left(\frac{\mathbf{j}}{4 n}\right)+12 \sum_{\mathbf{j} \in \Lambda^{f} \cap \mathbb{H}_{n}^{1,1}} c_{\mathbf{j}}^{(n)} f\left(\frac{\mathbf{j}}{4 n}\right) \\
& +12 \sum_{\mathbf{j} \in\left(\Lambda^{f} \cap \mathbb{H}_{n}^{1,2}\right) \cup\left(\Lambda^{f} \cap \mathbb{H}_{n}^{2,1}\right)} c_{\mathbf{j}}^{(n)} f\left(\frac{\mathbf{j}}{4 n}\right)+6 \sum_{\mathbf{j} \in \Lambda^{e, 1} \cap \mathbb{H}_{n}^{2,2}} c_{\mathbf{j}}^{(n)} f\left(\frac{\mathbf{j}}{4 n}\right) \\
& +4 \sum_{\mathbf{j} \in\left(\Lambda^{e, 2} \cap \mathbb{H}_{n}^{1,3}\right) \cup\left(\Lambda^{e, 2} \cap \mathbb{H}_{n}^{3,1}\right)} c_{\mathbf{j}}^{(n)} f\left(\frac{\mathbf{j}}{4 n}\right) \\
& =12 \sum_{\mathbf{j} \in \Lambda^{\circ} \cap \mathbb{H}_{n}^{1,1}} f\left(\frac{\mathbf{j}}{4 n}\right)+6 \sum_{\mathbf{j} \in \Lambda^{f} \cap \mathbb{H}_{n}^{1,1}} f\left(\frac{\mathbf{j}}{4 n}\right)+4 \sum_{\mathbf{j} \in\left(\Lambda^{f} \cap \mathbb{H}_{n}^{1,2}\right) \cup\left(\Lambda^{f} \cap \mathbb{H}_{n}^{2,1}\right)} f\left(\frac{\mathbf{j}}{4 n}\right) \\
& +\sum_{\mathbf{j} \in \Lambda^{e, 1} \cap \mathbb{H}_{n}^{2,2}} f\left(\frac{\mathbf{j}}{4 n}\right)+\sum_{\mathbf{j} \in\left(\Lambda^{e, 2} \cap \mathbb{H}_{n}^{1,3}\right) \cup\left(\Lambda^{e, 2} \cap \mathbb{H}_{n}^{3,1}\right)} f\left(\frac{\mathbf{j}}{4 n}\right) .
\end{aligned}
$$


Adding these two expressions together and using (4.10), we conclude that

$$
\begin{aligned}
& \sum_{\mathbf{j} \in \mathbb{H}_{n}^{*}} c_{\mathbf{j}}^{(n)} f\left(\frac{\mathbf{j}}{4 n}\right)=\sum_{\mathbf{j} \in \mathbb{H}_{n}^{\circ}} f\left(\frac{\mathbf{j}}{4 n}\right)+\sum_{\mathbf{j} \in \mathbb{H}_{n}^{*} \backslash \mathbb{H}_{n}^{\circ}} c_{\mathbf{j}}^{(n)} f\left(\frac{\mathbf{j}}{4 n}\right)=24 \sum_{\mathbf{j} \in \Lambda_{n}^{\circ}} f\left(\frac{\mathbf{j}}{4 n}\right) \\
& \quad+12 \sum_{\mathbf{j} \in \Lambda_{n}^{f}} f\left(\frac{\mathbf{j}}{4 n}\right)+6 \sum_{\mathbf{j} \in \Lambda_{n}^{e, 1}} f\left(\frac{\mathbf{j}}{4 n}\right)+4 \sum_{\mathbf{j} \in \Lambda_{n}^{e, 2}} f\left(\frac{\mathbf{j}}{4 n}\right)+\sum_{\mathbf{j} \in \Lambda_{n}^{v}} f\left(\frac{\mathbf{j}}{4 n}\right) \\
& =\sum_{\mathbf{j} \in \Lambda_{n}} \lambda_{\mathbf{j}}^{(n)} f\left(\frac{\mathbf{j}}{4 n}\right) .
\end{aligned}
$$

Replacing $f$ by $f \bar{g}$, we have proved that $\langle f, g\rangle_{n}^{*}=\langle f, g\rangle_{\triangle, n}$ whenever $f \bar{g}$ is invariant. Hence, (4.12) follows from Theorem 3.15, Furthermore, since $\frac{1}{\left|\Omega_{H}\right|} \int_{\Omega_{H}} f(\mathbf{t}) d \mathbf{t}$ $=\frac{1}{|\Delta|_{H}} \int_{\triangle_{H}} f(\mathbf{t}) d \mathbf{t}$ for all invariant $f$, (4.13) follows from Theorem 3.16

Furthermore, replacing $f$ by $\mathrm{TC}_{\mathbf{k}} \overline{\mathrm{TC}_{\mathbf{j}}}$ in (4.15), we derive by (4.3) that

$$
\begin{aligned}
& \left\langle\mathrm{TC}_{\mathbf{k}}, \mathrm{TC}_{\mathbf{j}}\right\rangle_{\triangle, n}=\frac{1}{4 n^{3}} \sum_{\mathbf{l} \in \mathbb{H}_{n}^{*}} c_{\mathbf{l}}^{(n)} \mathrm{TC}_{\mathbf{k}}\left(\frac{\mathbf{l}}{4 n}\right) \overline{\mathrm{TC}_{\mathbf{j}}\left(\frac{1}{4 n}\right)} \\
& =\frac{1}{4 n^{3}} \frac{1}{24} \sum_{\mathbf{l} \in \mathbb{H}_{n}^{*}} c_{\mathbf{l}}^{(n)} \sum_{\sigma \in \mathcal{G}} \phi_{\mathbf{k}}\left(\frac{\mathbf{l} \sigma}{4 n}\right) \overline{\mathrm{TC}_{\mathbf{j}}\left(\frac{1}{4 n}\right)}=\frac{1}{4 n^{3}} \frac{1}{24} \sum_{\mathbf{l} \in \mathbb{H}_{n}^{*}} c_{\mathbf{l}}^{(n)} \sum_{\sigma \in \mathcal{G}} \phi_{\mathbf{k}}\left(\frac{\mathbf{l} \sigma}{4 n}\right) \overline{\mathrm{TC}_{\mathbf{j}}\left(\frac{1 \sigma}{4 n}\right)} \\
& =\frac{1}{4 n^{3}} \frac{|\mathcal{G}|}{24} \sum_{\mathbf{l} \in \mathbb{H}_{n}^{*}} c_{\mathbf{l}}^{(n)} \phi_{\mathbf{k}}\left(\frac{1}{4 n}\right) \overline{\mathrm{TC}_{\mathbf{j}}\left(\frac{1}{4 n}\right)}=\left\langle\phi_{\mathbf{k}}, \mathrm{TC}_{\mathbf{j}}\right\rangle_{n}^{*}=\frac{1}{24} \sum_{\sigma \in \mathcal{G}}\left\langle\phi_{\mathbf{k}}, \phi_{\mathbf{j} \sigma}\right\rangle_{n}^{*} .
\end{aligned}
$$

Using (3.25) and abbreviating $\mathbf{k} \equiv \mathbf{j} \bmod 4 \mathbb{Z}_{\mathbb{H}}^{4}$ as $\mathbf{k} \equiv \mathbf{j}$, we further deduce that

$$
\left\langle\mathrm{TC}_{\mathbf{k}}, \mathrm{TC}_{\mathbf{j}}\right\rangle_{\triangle, n}=\frac{1}{24}|\{\sigma \in \mathcal{G}: \mathbf{j} \sigma \equiv \mathbf{k}\}|=\frac{\delta_{\mathbf{j}, \mathbf{k}}}{24}|\{\sigma \in \mathcal{G}: \mathbf{k} \sigma \equiv \mathbf{k}\}|=\frac{\delta_{\mathbf{j}, \mathbf{k}}}{\lambda_{\mathbf{k}}^{(n)}}
$$

where the last equality follows from a direct counting.

The proof of the above theorem also applies to $f, g \in \mathcal{T} S_{n}$ since $f \bar{g}$ is invariant if both $f$ and $g$ are anti-invariant. Moreover, $f \bar{g} \in \mathcal{T}_{2 n-1}$ if $f, g \in \mathcal{T}_{n}$. Notice also that $\operatorname{TS}_{\mathbf{k}}\left(\frac{\mathbf{j}}{4 n}\right)=0$ when $\mathbf{j} \in \Lambda_{n} \backslash \Lambda_{n}^{\circ}$, we deduce the following result.

Theorem 4.5. Let the discrete inner product $\langle\cdot, \cdot\rangle_{\triangle^{\circ}, n}$ be defined by

$$
\langle f, g\rangle_{\triangle^{\circ}, n}=\frac{6}{n^{3}} \sum_{\mathbf{j} \in \Lambda_{n}^{\circ}} f\left(\frac{\mathbf{j}}{4 n}\right) \overline{g\left(\frac{\mathbf{j}}{4 n}\right)} .
$$

Then

$$
\langle f, g\rangle_{\triangle^{\circ}, n}=\langle f, g\rangle_{\triangle_{H}}, \quad f, g \in \mathcal{T} S_{n} .
$$

4.3. Interpolation on the tetrahedron. We can deduce results on interpolation on the tetrahedron by making use of the orthogonality of generalized trigonometric functions with respect to the discrete inner product, as shown in our first result below. Recall the operator $\mathcal{P}^{ \pm}$defined in (4.1).

Theorem 4.6. For $n>0$, and $f \in C\left(\Delta_{H}\right)$, define

$$
\mathcal{L}_{n} f(\mathbf{t}):=\sum_{\mathbf{j} \in \Lambda_{n}^{\circ}} f\left(\frac{\mathbf{j}}{4 n}\right) \ell_{\mathbf{j}, n}^{\circ}(\mathbf{t}), \quad \ell_{\mathbf{j}, n}^{\circ}(\mathbf{t}):=\frac{144}{n^{3}} \sum_{\mathbf{k} \in \Lambda_{n}^{\circ}} \operatorname{TS}_{\mathbf{k}}(\mathbf{t}) \overline{\operatorname{TS}_{\mathbf{k}}\left(\frac{\mathbf{j}}{4 n}\right)} .
$$

Then $\mathcal{L}_{n} f$ is the unique function in $\mathcal{T} S_{n}$ that satisfies

$$
\mathcal{L}_{n} f\left(\frac{\mathbf{j}}{4 n}\right)=f\left(\frac{\mathbf{j}}{4 n}\right), \quad \mathbf{j} \in \Lambda_{n}^{\circ} .
$$


Furthermore, the fundamental interpolation function $\ell_{\mathbf{j}, n}^{\circ}$ is real and satisfies

$$
\ell_{\mathbf{j}, n}^{\circ}(\mathbf{t})=\frac{6}{n^{3}} \mathcal{P}_{\mathbf{t}}^{-}\left[\Theta_{n}\left(\mathbf{t}-\frac{\mathbf{j}}{4 n}\right)-\Theta_{n-1}\left(\mathbf{t}-\frac{\mathbf{j}}{4 n}\right)\right],
$$

where $\mathcal{P}_{\mathbf{t}}^{-}$means that the operator $\mathcal{P}^{-}$is acting on the variable $\mathbf{t}$ and $\Theta_{n}$ is defined in (3.18).

Proof. By (4.6), $\left\langle\mathrm{TS}_{\mathbf{j}}, \mathrm{TS}_{\mathbf{k}}\right\rangle_{\triangle^{\circ}, n}=\frac{1}{24} \delta_{\mathbf{j}, \mathbf{k}}$, which shows that $\ell_{\mathbf{j}, n}^{\circ}\left(\frac{\mathbf{k}}{4 n}\right)=\delta_{\mathbf{j}, \mathbf{k}}$ and verifies the interpolation condition. It follows from the definition of $\mathrm{TS}_{\mathbf{k}}$ that

$$
\ell_{\mathbf{j}, n}^{\circ}(\mathbf{t})=\frac{6}{n^{3}} \mathcal{P}_{\mathbf{t}}^{-} \mathcal{P}_{\mathbf{j}}^{-} \sum_{\mathbf{k} \in \mathbb{H}_{n}^{\circ}} \phi_{\mathbf{k}}(\mathbf{t}) \overline{\phi_{\mathbf{k}}\left(\frac{\mathbf{j}}{4 n}\right)}
$$

Furthermore, we can replace the summation over $\mathbf{k} \in \mathbb{H}_{n}^{\circ}$ by the summation over $\mathbf{k} \in \mathbb{H}_{n}$ since $\mathrm{TS}_{\mathbf{k}}\left(\frac{\mathbf{j}}{4 n}\right)=\mathrm{TS}_{\mathbf{j}}\left(\frac{\mathbf{k}}{4 n}\right)=0$ whenever $\mathbf{k} \in \Lambda_{n} \backslash \Lambda_{n}^{\circ}$, and $\mathrm{TS}_{\mathbf{k}}(\mathbf{t})=0$ whenever at least two components of $\mathbf{k}$ are equal. Consequently, we conclude that

$$
\ell_{\mathbf{j}, n}^{\circ}(\mathbf{t})=\frac{6}{n^{3}} \mathcal{P}_{\mathbf{t}}^{-} \mathcal{P}_{\mathbf{j}}^{-} D_{n}^{H}\left(\mathbf{t}-\frac{\mathbf{j}}{4 n}\right),
$$

where $D_{n}^{H}$ is the Dirichlet kernel for the rhombic dodecahedral Fourier partial sum defined in (3.17). Recall that $\mathcal{G}$ is a permutation group and $|\sigma|$ denotes the number of inversions in $\sigma \in \mathcal{G}$. Let $f$ be an invariant function under $\mathcal{G}$. Then

$$
\begin{aligned}
\mathcal{P}_{\mathbf{t}}^{-} \mathcal{P}_{\mathbf{s}}^{-} f(\mathbf{t}-\mathbf{s}) & =\frac{1}{|\mathcal{G}|^{2}} \sum_{\sigma \in \mathcal{G}} \sum_{\tau \in \mathcal{G}}(-1)^{|\sigma|+|\tau|} f(\mathbf{t} \sigma-\mathbf{s} \tau) \\
& =\frac{1}{|\mathcal{G}|^{2}} \sum_{\tau \in \mathcal{G}} \sum_{\sigma \in \mathcal{G}}(-1)^{|\sigma|+|\tau|} f\left(\mathbf{t} \sigma \tau^{-1}-\mathbf{s}\right) \\
& =\frac{1}{|\mathcal{G}|} \sum_{\sigma \in \mathcal{G}}(-1)^{|\sigma|} f(\mathbf{t} \sigma-\mathbf{s})=\mathcal{P}_{\mathbf{t}}^{-} f(\mathbf{t}-\mathbf{s}),
\end{aligned}
$$

where in the third equal sign we have used the fact that $|\sigma \tau|+|\tau|=|\sigma|$, which can be easily verified. Setting $f=D_{n}^{H}$ completes the proof.

The function $\mathcal{L}_{n} f$ interpolates at the interior points of $\Lambda_{n}$. We can also derive an analog result for interpolation on $\Lambda_{n}$ by using the same approach. However, it is more illustrating to derive it from the interpolation on the rhombic dodecahedron, which we carry out below.

Theorem 4.7. For $n>0$ and $f \in C\left(\Delta_{H}\right)$ define

$$
\mathcal{L}_{n}^{*} f(\mathbf{t}):=\sum_{\mathbf{j} \in \Lambda_{n}} f\left(\frac{\mathbf{j}}{4 n}\right) \ell_{\mathbf{j}, n}^{\Delta}(\mathbf{t}), \quad \ell_{\mathbf{j}, n}^{\triangle}(\mathbf{t}):=\frac{\lambda_{\mathbf{j}}^{(n)}}{4 n^{3}} \sum_{\mathbf{k} \in \Lambda_{n}} \lambda_{\mathbf{k}}^{(n)} \mathrm{TC}_{\mathbf{k}}(\mathbf{t}) \overline{\mathrm{TC}_{\mathbf{k}}\left(\frac{\mathbf{j}}{4 n}\right)} .
$$

Then $\mathcal{L}_{n}^{*} f$ is the unique function in $\mathcal{T} C_{n}$ that satisfies

$$
\mathcal{L}_{n}^{*} f\left(\frac{\mathbf{j}}{4 n}\right)=f\left(\frac{\mathbf{j}}{4 n}\right), \quad \mathbf{j} \in \Lambda_{n} .
$$

Furthermore, the fundamental interpolation function $\ell_{\mathbf{j}, n}^{\triangle}$ is given by

$$
\ell_{\mathbf{j}, n}^{\Delta}(\mathbf{t})=\lambda_{\mathbf{j}}^{(n)} \mathcal{P}^{+} \ell_{\mathbf{j}, n}(\mathbf{t}) .
$$


Proof. It follows from (4.14) that $\ell_{\mathbf{j}, n}^{\triangle}\left(\frac{\mathbf{k}}{4 n}\right)=\delta_{\mathbf{k}, \mathbf{j}}$ for $\mathbf{k}, \mathbf{j} \in \Lambda_{n}$, which verifies the interpolation condition. Furthermore, in the proof of Theorem 4.4, we established that $\sum_{\mathbf{j} \in \Lambda_{n}} \lambda_{\mathbf{j}}^{(n)} g\left(\frac{\mathbf{j}}{4 n}\right)=\sum_{j \in \mathbb{H}_{n}^{*}} c_{\mathbf{j}}^{(n)} g\left(\frac{\mathbf{j}}{4 n}\right)$ for function $g$ invariant under $\mathcal{G}$. Applying this relation to $g\left(\frac{\mathbf{k}}{4 n}\right)=\mathrm{TC}_{\mathbf{k}}(\mathbf{t}) \mathrm{TC}_{\mathbf{k}}\left(\frac{\mathbf{j}}{4 n}\right)$, we obtain

$$
\begin{aligned}
\ell_{\mathbf{j}, n}^{\triangle}(\mathbf{t}) & =\frac{\lambda_{\mathbf{j}}^{(n)}}{4 n^{3}} \sum_{\mathbf{k} \in \mathbb{H}_{n}^{*}} c_{\mathbf{k}}^{(n)} \mathrm{TC}_{\mathbf{k}}(\mathbf{t}) \overline{\operatorname{TC}_{\mathbf{k}}\left(\frac{\mathbf{j}}{4 n}\right)} \\
& =\frac{\lambda_{\mathbf{j}}^{(n)}}{4 n^{3}} \mathcal{P}_{\mathbf{t}}^{+} \mathcal{P}_{\mathbf{j}}^{+} \sum_{\mathbf{k} \in \mathbb{H}_{n}^{*}} c_{\mathbf{k}}^{(n)} \phi_{\mathbf{k}}(\mathbf{t}) \overline{\phi_{\mathbf{k}}\left(\frac{\mathbf{j}}{4 n}\right)}=\lambda_{\mathbf{j}}^{(n)} \mathcal{P}_{\mathbf{t}}^{+} \mathcal{P}_{\mathbf{j}}^{+} \Phi_{n}^{*}\left(\mathbf{t}-\frac{\mathbf{j}}{4 n}\right) .
\end{aligned}
$$

Using the fact that $\mathcal{G}$ is a permutation group, it is easy to see that

$$
\mathcal{P}_{\mathbf{t}}^{+} \mathcal{P}_{\mathbf{s}}^{+} f(\mathbf{t}-\mathbf{s})=\mathcal{P}_{\mathbf{t}}^{+} f(\mathbf{t}-\mathbf{s}),
$$

for an invariant function $f$. Consequently,

$$
\ell_{\mathbf{j}, n}^{\triangle}(\mathbf{t})=\lambda_{\mathbf{j}}^{(n)} \mathcal{P}_{\mathbf{t}}^{+} \Phi_{n}^{*}\left(\mathbf{t}-\frac{\mathbf{j}}{4 n}\right)=\lambda_{\mathbf{j}}^{(n)} \mathcal{P}^{+} \ell_{\mathbf{j}, n}(\mathbf{t}) .
$$

The proof is completed.

Recall the explicit formula of $\ell_{\mathbf{j}, n}$ given in Theorem $3.18, \ell_{j, n}^{\triangle}$ enjoys a compact formula.

Let $\left\|\mathcal{L}_{n}\right\|$ and $\left\|\mathcal{L}_{n}^{*}\right\|$ denote the operator norms of $\mathcal{L}_{n}$ and $\mathcal{L}_{n}^{*}$, respectively, both as operators from $C\left(\triangle_{H}\right) \mapsto C\left(\triangle_{H}\right)$. From Theorems 4.6 and 4.7, an immediate application of Theorem 3.19 yields the following theorem.

Theorem 4.8. There is a constant $c$ independent of $n$, such that

$$
\left\|\mathcal{L}_{n}\right\| \leq c(\log n)^{3} \quad \text { and } \quad\left\|\mathcal{L}_{n}^{*}\right\| \leq c(\log n)^{3} .
$$

4.4. Interpolation on the regular tetrahedron. The results in the above are developed in homogeneous coordinates. Here we indicate how they can be recast into the usual coordinates on the regular tetrahedron $\triangle^{*}$ defined by

$$
\triangle^{*}:=\left\{x \in \mathbb{R}^{3}: 0 \leq x_{3} \leq x_{2} \leq x_{1} \leq 1\right\}
$$

as depicted in the Figure 4.3 below.

The change of variable from $\mathbf{t}$ to $x \in \mathbb{R}^{3}$ is given in (3.2). When we transform the formulas from the homogeneous coordinates to the regular coordinates, we also need to transform the indices from $\mathbf{j} \in \mathbb{Z}_{H}^{4}$ to $\mathbb{Z}^{3}$ by using

$$
k=\frac{1}{4} A^{\operatorname{tr}} U^{\operatorname{tr}} \mathbf{j} \Longleftrightarrow\left\{\begin{array}{l}
k_{1}=\frac{1}{4}\left(j_{1}-j_{4}\right), \\
k_{2}=\frac{1}{4}\left(j_{2}-j_{4}\right), \\
k_{3}=\frac{1}{4}\left(j_{3}-j_{4}\right) .
\end{array}\right.
$$

Under this change of variables, it is easy to see that the point set $\Lambda_{n}$ becomes $\left\{k \in \mathbb{Z}^{3}: 0 \leq k_{3} \leq k_{2} \leq k_{1} \leq n\right\}$. For example, the cubature formula in (4.13) becomes the following:

Theorem 4.9. For $n>0$, the cubature formula

$$
6 \int_{\triangle^{*}} f\left(x_{1}, x_{2}, x_{3}\right) d x_{1} d x_{2} d x_{3}=\frac{1}{4 n^{3}} \sum_{0 \leq k_{3} \leq k_{2} \leq k_{1} \leq n} \lambda_{k_{1}, k_{2}, k_{3}}^{(n)} f\left(\frac{k_{1}}{n}, \frac{k_{2}}{n}, \frac{k_{3}}{n}\right)
$$

is exact for all $f \in \mathcal{T} C_{2 n-1}$, where $\lambda_{k_{1}, k_{2}, k_{3}}^{(n)}=\lambda_{\mathbf{j}}^{(n)}$ with $\mathbf{j}$ given by (4.16). 


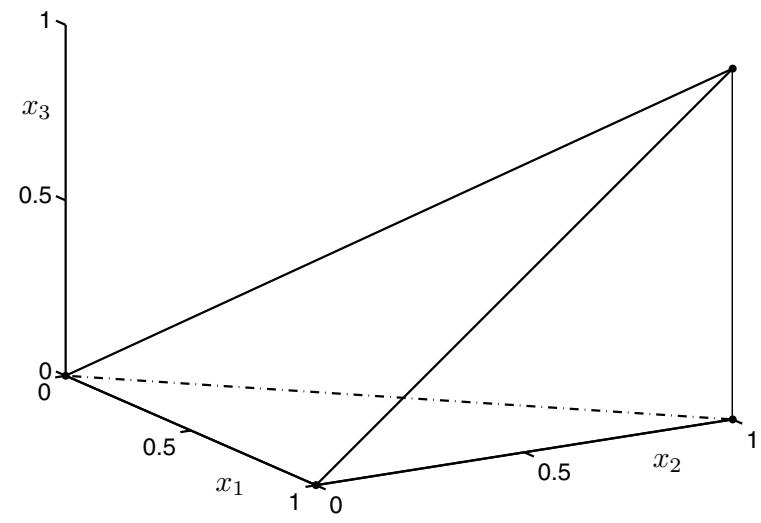

FigURE 4.3. Regular tetrahedron

We also note that the Dirichlet kernel in (3.18) can be recast into $x$ coordinates by (3.2) straightforwardly, so is the fundamental interpolation function $\ell_{\mathbf{j}, n}(\mathbf{t})$ given in Theorem 3.18. Consequently, the Lagrange interpolation function in Theorem 4.7 becomes

$$
\mathcal{L}_{n}^{*} f(x)=\sum_{0 \leq k_{3} \leq k_{2} \leq k_{1} \leq n} f\left(\frac{k_{1}}{n}, \frac{k_{2}}{n}, \frac{k_{3}}{n}\right) \ell_{k, n}^{\triangle}(x),
$$

where $\ell_{k, n}^{\triangle}$ satisfies the compact formula

$$
\ell_{k, n}^{\triangle}(x)=\lambda_{k}^{(n)} \mathcal{P}^{+} \ell_{\mathbf{j}, n}(\mathbf{t}), \quad \text { where } \quad \ell_{\mathbf{j}, n}(\mathbf{t})=\Phi_{n}^{*}\left(\mathbf{t}-\frac{\mathbf{j}}{4 n}\right)
$$

with $\Phi_{n}^{*}$ given in (3.24),$\lambda_{k}^{(n)}=\lambda_{\mathbf{j}}^{(n)}$ with $\mathbf{j}$ as in (4.16). In the above formula we apply $\mathcal{P}^{+}$to the compact formula of $\Phi_{n}^{*}$ first and then use (3.2) to change from $\mathbf{t}$ to $x$.

\section{ACKNOWLEDGMENT}

The authors thank an anonymous referee for his careful reading and invaluable comments.

\section{REFERENCES}

[1] L. Bos, Bounding the Lebesgue function for Lagrange interpolation in a simplex, J. Approx. Theory 38 (1983), 43-59. MR700876 (84e:41007)

[2] J. H. Conway and N. J. A. Sloane, Sphere Packings, Lattices and Groups, 3rd ed. Springer, New York, 1999. MR1662447 (2000b:11077)

[3] D. E. Dudgeon and R. M. Mersereau, Multidimensional Digital Signal Processing, PrenticeHall. Inc., Englewood Cliffs, New Jersey, 1984.

[4] C. F. Dunkl and Yuan Xu, Orthogonal polynomials of several variables, Encyclopedia of Mathematics and its Applications, vol. 81, Cambridge Univ. Press, 2001. MR.1827871 (2002m:33001)

[5] B. Fuglede, Commuting self-adjoint partial differential operators and a group theoretic problem, J. Functional Anal. 16 (1974), 101-121. MR0470754 (57:10500)

[6] T. C. Hales, A proof of the Kepler conjecture. Ann. of Math. (2) 162 (2005), no. 3, 1065-1185. MR2179728 (2006g:52029)

[7] J. R. Higgins, Sampling theory in Fourier and Signal Analysis, Foundations, Oxford Science Publications, New York, 1996. 
[8] T. Koornwinder, Orthogonal polynomials in two variables which are eigenfunctions of two algebraically independent partial differential operators, Nederl. Acad. Wetensch. Proc. Ser. A77 = Indag. Math. 36 (1974), 357-381. MR0357905 (50:10371a)

[9] H. Li, J. Sun and Yuan Xu, Discrete Fourier analysis, cubature and interpolation on a hexagon and a triangle, SIAM J. Numer. Anal. 46 (2008), 1653-1681. MR2399390

[10] R. J. Marks II, Introduction to Shannon Sampling and Interpolation Theory, Springer-Verlag, New York, 1991. MR.1077829 (92j:41001)

[11] J. Sun, Multivariate Fourier series over a class of non tensor-product partition domains, J. Comput. Math. 21 (2003), 53-62. MR.1974272 (2004m:42011)

[12] J. Sun, Multivariate Fourier transform methods over simplex and super-simplex domains, J. Comput. Math. 24 (2006), 305-322. MR2229712(2007a:42019)

[13] A. Zygmund, Trigonometric series, Cambridge Univ. Press, Cambridge, 1959. MR0107776 $(21: 6498)$

Institute of Software, Chinese Academy of Sciences, Beijing 100190, China

E-mail address: hynli@mail.rdcps.ac.cn

Department of Mathematics, University of Oregon, Eugene, Oregon 97403-1222.

E-mail address: yuan@math.uoregon.edu 